ARTICLE

Received 25 Feb 2014 | Accepted 16 Apr 2014 | Published 4 Jun $2014 \quad$ DOl: 10.1038/ncomms4917

\title{
The FAM3 superfamily member ILEI ameliorates Alzheimer's disease-like pathology by destabilizing the penultimate amyloid- $\beta$ precursor
}

Hiroshi Hasegawa ${ }^{1, \star, \dagger}$, Lei Liu ${ }^{1, \star}$, Ikuo Tooyama${ }^{1}$, Shigeo Murayama ${ }^{2} \&$ Masaki Nishimura ${ }^{1}$

Accumulation of amyloid- $\beta$ peptide $(A \beta)$ in the brain underlies the pathogenesis of Alzheimer's disease (AD). A $\beta$ is produced by $\beta$ - and $\gamma$-secretase-mediated sequential proteolysis of amyloid- $\beta$ precursor protein (APP). Here we identify a secretory protein named interleukinlike epithelial-mesenchymal transition inducer (ILEI, also known as FAM3 superfamily member $C$ ) as a negative regulator of $A \beta$ production. ILEI destabilizes the $\beta$-secretasecleaved APP carboxy-terminal fragment, the penultimate precursor of $A \beta$, by binding to the $\gamma$-secretase complex and interfering with its chaperone properties. Notch signalling and $\gamma$-secretase activity are not affected by ILEI. We also show neuronal expression of ILEI and its induction by transforming growth factor- $\beta$ signalling. The level of secreted ILEI is markedly decreased in the brains of AD patients. Transgenic ( $\mathrm{Tg}$ ) overexpression of ILEI significantly reduces the brain $A \beta$ burden and ameliorates the memory deficit in $A D$ model mice. ILEl may be a plausible target for the development of disease-modifying therapies.

\footnotetext{
${ }^{1}$ Molecular Neuroscience Research Center, Shiga University of Medical Science, Seta-Tsukinowa, Otsu, Shiga 520-2192, Japan. ${ }^{2}$ Department of Neuropathology, Tokyo Metropolitan Institute of Gerontology, Tokyo 173-0015, Japan. * These authors contributed equally to this work. $\dagger$ Present address: Minami-Kyoto Hospital, Kyoto, Japan. Correspondence and requests for materials should be addressed to M.N. (e-mail: mnishimu@belle.shiga-med.ac.jp).
} 
$\mathrm{T}$ he amyloid- $\beta$ peptide $(A \beta)$ is produced in neurons by two-step proteolytic processing of the amyloid- $\beta$ precursor protein $(\mathrm{APP})^{1}$. Ectodomain shedding by $\beta$-secretase, which is known as $\beta$-site APP-cleaving enzyme 1 (BACE1), yields the membrane-spanning carboxy-terminal fragment (CTF)- $\beta$. The aspartyl protease $\gamma$-secretase complex, which primarily comprises presenilin, nicastrin (NCT), anterior pharynx defective-1 (APH-1) and presenilin enhancer-2 (PEN-2), sequentially catalyses intramembrane proteolysis of APP-CTF $\beta$, resulting in secretion of $A \beta$ and liberation of the APP intracellular domain ${ }^{1}$. Each proteolysed derivative exhibits distinct physiological activities ${ }^{2}$, and genetic ablation of APP in mice causes neurological deficits such as impairment of spatial learning and reduction in brain weight, which can be completely rescued by expression of the secreted ectodomain ${ }^{3}$. Ablation of the $\gamma$-secretase complex by genetic deletion of any of the core components causes early developmental defects that resemble a Notch phenotype, which is expected because $\gamma$-secretase is involved in transmembrane signal transduction by cleaving various membrane-spanning substrates, including Notch receptors ${ }^{1}$.

A persistent imbalance between production and clearance of $A \beta$ results in gradual accumulation of soluble and aggregated $A \beta$ in the extracellular space of the brain parenchyma, which underlies the development of Alzheimer's disease (AD). Genetic mutations in APP, presenilin-1 (PS1) and presenilin-2 (PS2) modify A $\beta$ biosynthesis and cause an autosomal dominant form of $\mathrm{AD}^{4}$. In contrast, alteration in $\mathrm{A} \beta$ metabolism in the common sporadic form of $\mathrm{AD}$ has not been fully clarified. However, an increase in the amount and activity of BACE1 and/ or a decline in protein degradation capacity have been proposed as the primary events in the pathogenesis of sporadic $\mathrm{AD}^{5-8}$. In addition to $A \beta$, accumulation of APP-CTFs, which are sometimes associated with sporadic $\mathrm{AD}$, may cause neurodegeneration by inducing abnormal membrane currents, decreased numbers of dendritic spines, neuronal cell death and inflammatory reactions ${ }^{9,10}$.

Reduction in brain $A \beta$ is the pivotal goal of disease-modifying therapy for $\mathrm{AD}$. Although $\gamma$-secretase is a major target for therapeutic intervention, non-selective inhibition of its activity causes serious adverse effects due to blockade of Notch signalling and accumulation of neurotoxic APP-CTFs ${ }^{1,11-13}$. To avoid these adverse effects, finding novel approaches to negatively modulate $A \beta$ generation is imperative. Several endogenous proteins interact with the $\gamma$-secretase complex and influence the efficacy of $\gamma$-secretase cleavage in distinct ways, including alteration of specific activities, substrate selectivity and assembly or subcellular localization of $\gamma$-secretase complexes ${ }^{14-17}$.

In this study, we searched for additional proteins that affect $A \beta$ generation by interacting with the $\gamma$-secretase complex. To date, multiple $\gamma$-secretase-modulating proteins have been identified by purification of intact $\gamma$-secretase complexes via affinity-mediated isolation of the major catalytic component, PS1 (refs 18,19), which is also involved in cellular $\mathrm{Ca}$ homeostasis, apoptosis and protein trafficking by binding additional or alternative partners ${ }^{14}$. In contrast, PEN-2 is exclusively involved in formation of the $\gamma$-secretase complex, as the cellular accumulation of PEN-2 is entirely dependent on the expression of the other core components. In addition, $\mathrm{PEN}-2$ is the last component to be incorporated into the $\gamma$-secretase complex, and activated complexes exclusively contain PEN-2. Here, using PEN-2 affinity-mediated isolation of the $\gamma$-secretase complex, we identify a unique secretory protein that reduces cellular $A \beta$ generation without inhibiting $\gamma$-secretase activity or Notch cleavage.

\section{Results}

ILEI is a $\gamma$-secretase complex-binding protein. We employed tandem affinity-tag purification (TAP) ${ }^{20}$ to isolate the $\gamma$-secretase complex using PEN-2 as the bait, combined with stable silencing of endogenous PEN-2 expression. The abundance and stoichiometry of $\gamma$-secretase components are tightly regulated. Although exogenous PEN-2 can be incorporated into the $\gamma$-secretase complex by replacing endogenous PEN-2, high PEN-2 overexpression is required for sufficient replacement. To circumvent this problem, we first prepared PEN-2-knockdown HEK293 cell lines in which small interference RNA (siRNA) for PEN-2 was stably expressed. We chose a PEN-2-knockdown cell line that exhibited the lowest levels of PEN-2 expression and A $\beta$ secretion. We then transfected this cell line with siRNA-resistant PEN-2 (srPEN-2) fused with a TAP tag (TAP-srPEN-2) and selected a cell line, referred to as HEK-TAP-PEN-2, which stably expressed TAP-srPEN-2 at a level equivalent to that of endogenous PEN-2. We confirmed that TAP-srPEN-2 was incorporated into the active $\gamma$-secretase complex and restored $A \beta$ secretion (Supplementary Fig. 1).

The activity and composition of $\gamma$-secretase complexes are sensitive to several detergents but are only slightly affected by $1 \%$ 3-[(3-cholamidopropyl)dimethylammonio]-2-hydroxy-1-propanesulfonic acid (CHAPSO) ${ }^{21}$. We prepared CHAPSO lysates of HEK-TAP-PEN-2 cells and performed sequential isolation of TAP-srPEN-2-associated complexes. To distinguish non-specific binding proteins from proteins of interest, we used TAP-tagtransfected PEN-2-knockdown HEK293 (HEK-TAP-empty) cells as a control. Complexes isolated in two independent trials exhibited a similar pattern of banding on SYPRO- and silverstained SDS-polyacrylamide gel electrophoresis (SDS-PAGE) gels (Fig. 1a). Proteins in each excised gel piece were digested with trypsin and subjected to liquid chromatography-tandem mass spectrometric analysis followed by a search of the Mascot database. Hits included the four core components and some known binding proteins of the $\gamma$-secretase complex. One of the identified peptides matched residues $164-179$ of a $27-\mathrm{kDa}$ protein referred to as interleukin-like epithelial-mesenchymal transition inducer (ILEI) or family with sequence similarity 3 , member C (FAM3C) (NCBI Protein Database accession code NP_055703).

ILEI, which was originally identified in a database search for four-helix-bundle cytokines, is an evolutionarily conserved secretory protein ${ }^{22}$. ILEI is posttranslationally liberated from the membrane in secretory vesicles by cleavage of the signal sequence of the full-length (FL) precursor protein. Immunoblotting with an affinity-purified rabbit polyclonal antiILEI antibody revealed specific bands of 24 and $27 \mathrm{kDa}$ in the conditioned medium and cell lysate of HEK293 cells, respectively, suggesting that the smaller species corresponded to a processed, secreted form of ILEI (Fig. 1b). Co-immunoprecipitation using native HEK293 cells indicated that antibodies against PS1, NCT, PEN-2 or APH-1 co-precipitated the precursor and processed forms of ILEI (Fig. 1b). The reverse assay revealed that the antiILEI antibody co-precipitated the four core components of the $\gamma$ secretase complex (Fig. 1c). Protein complex analysis using twodimensional Blue Native SDS-PAGE suggested that ILEI is incorporated into multiple complexes of various molecular masses, one of which is comparable to that of the mature $\gamma$ secretase complex (Fig. 1d). These data confirmed the interaction between ILEI and the $\gamma$-secretase complex.

To determine the partner that directly interacts with endogenous ILEI, we treated HEK293 cells in situ with a thiolcleavable, membrane-permeable, chemical crosslinker. The cells were then lysed in a Nonidet P-40 buffer to dissociate noncrosslinked components of the $\gamma$-secretase complex. In this lysate, monomeric components of the $\gamma$-secretase complex were reduced 
a

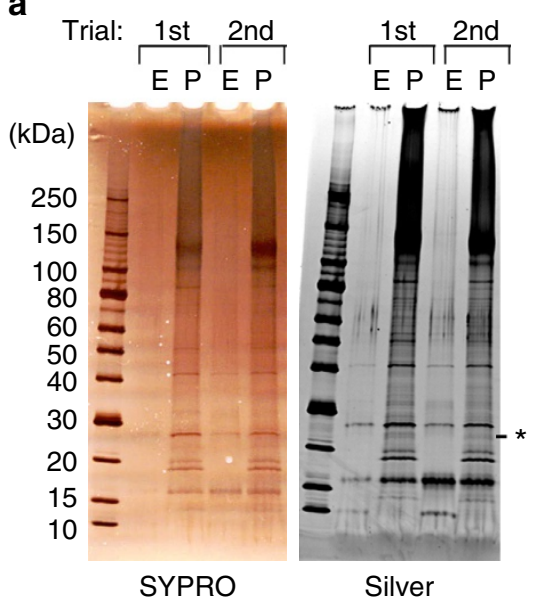

b

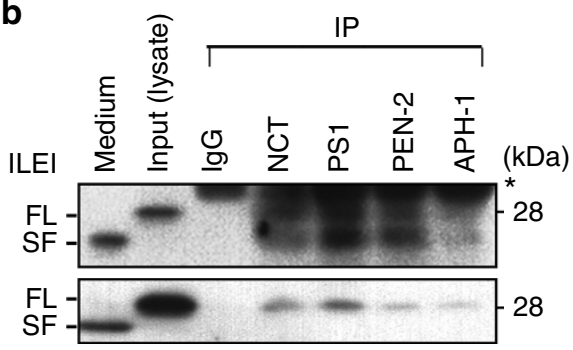

d

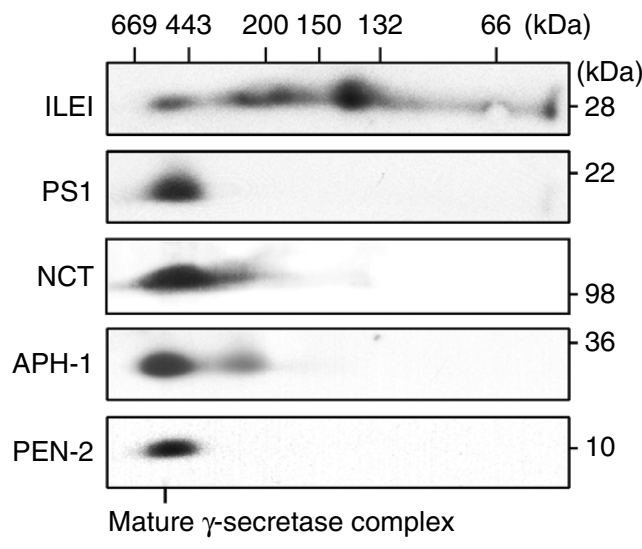

e

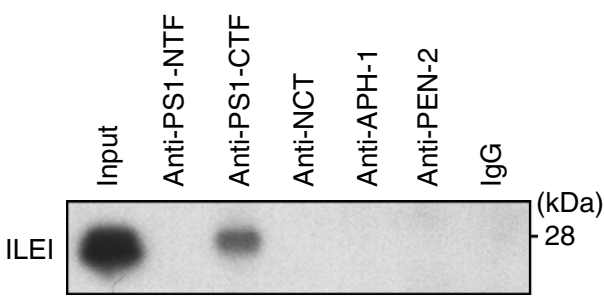

C

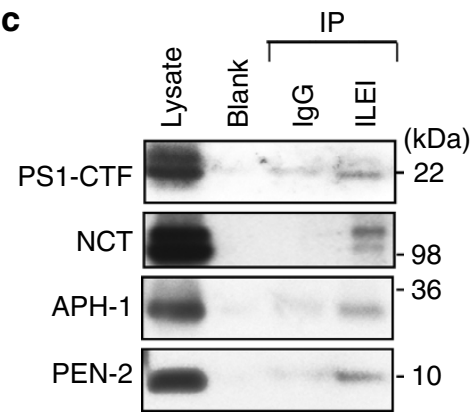

f

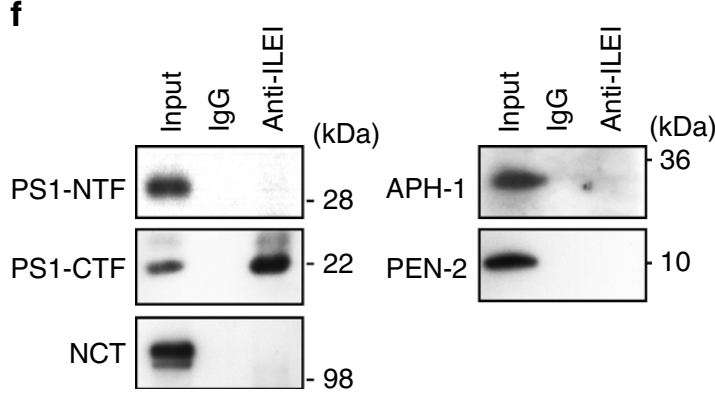

Figure 1 | ILEI is associated with mature $\boldsymbol{\gamma}$-secretase complexes. (a) SYPRO- and silver-stained SDS-PAGE gels of TAP-tagged PEN-2-associated proteins isolated from lysates of HEK-TAP-PEN-2 (P) and HEK-TAP-empty (E) cells. ILEl was identified in the band indicated by the star. (b) Coimmunoprecipitation of endogenous ILEI and $\gamma$-secretase components. Native HEK293 cell lysates were immunoprecipitated with normal lgG or an antibody against NCT, PS1, PEN-2 or APH-1. The blot was probed with anti-ILEl antibody (upper panel). As the FL ILEI bands were close to the IgG light-chain bands, nanocapsules incorporating IgG Fc-binding Z domains were used instead of the secondary antibody for detection (lower panel). The star indicates the IgG band. FL, full-length; SF, secreted form. (c) Reverse co-immunoprecipitation. HEK293 cell lysates were precipitated using normal rabbit IgG (IP-IgG) or anti-ILEI antibody (IP-ILEI). The blots were probed using the indicated antibodies. (d) Two-dimensional Blue Native (BN)/SDS-PAGE analysis of ILEI and $\gamma$-secretase components. The membrane fraction of native HEK293 cells was subjected to two-dimensional BN/SDS-PAGE before immunoblotting. (e) Chemical crosslinking of ILEI with $\gamma$-secretase components. HEK293 cells were crosslinked in situ with dithiobis succinimidyl propionate. The $1 \%$ Nonidet P-40-solubilized cell lysate was subjected to immunoprecipitation. A blot of the immunoprecipitates with antibodies against the indicated proteins or normal IgG was probed with anti-ILEl antibody. (f) The crosslinked cell lysates were immunoprecipitated using normal lgG or anti-ILEI antibody. The blots were probed using the indicated antibodies.

by $>80 \%$ on immunoblotting. PS1-CTF but not the other components co-immunoprecipitated ILEI (Fig. 1e). Conversely, the ILEI immunoprecipitates contained PS1-CTF but the other components were not present or were only faintly observed (Fig. 1f). These results suggest that ILEI directly binds to PS1-CTF.

ILEI reduces $\mathbf{A} \boldsymbol{\beta}$ generation by destabilizing APP-CTF $\beta$. RNA interference (RNAi)-mediated knockdown of endogenous ILEI in native HEK293 cells resulted in an approximately two-fold increase in $A \beta 40$ and $A \beta 42$ levels in the conditioned medium (Fig. 2a). Conversely, overexpression of ILEI decreased $A \beta$ secretion (Fig. 2b). No significant alteration in the ratio of $A \beta 40$ to $A \beta 42$ was observed. ILEI knockdown or overexpression also increased or decreased APP intracellular domain generation, respectively (Fig. 2c).

We next determined whether ILEI affects $\gamma$-secretase cleavage of the Notch-1 receptor. Treatment with EDTA enhances the site2 cleavage of transmembrane/intracellular Notch to generate Notch extracellular truncation, which is then directly cleaved by $\gamma$-secretase to release Notch intracellular domain ${ }^{23}$. ILEI 
a

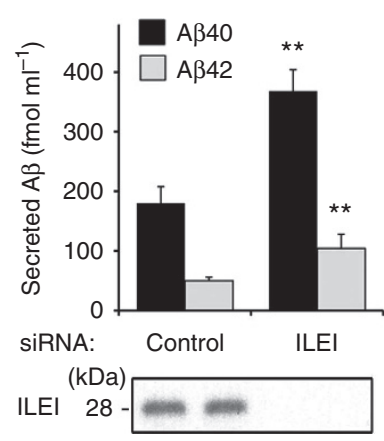

C
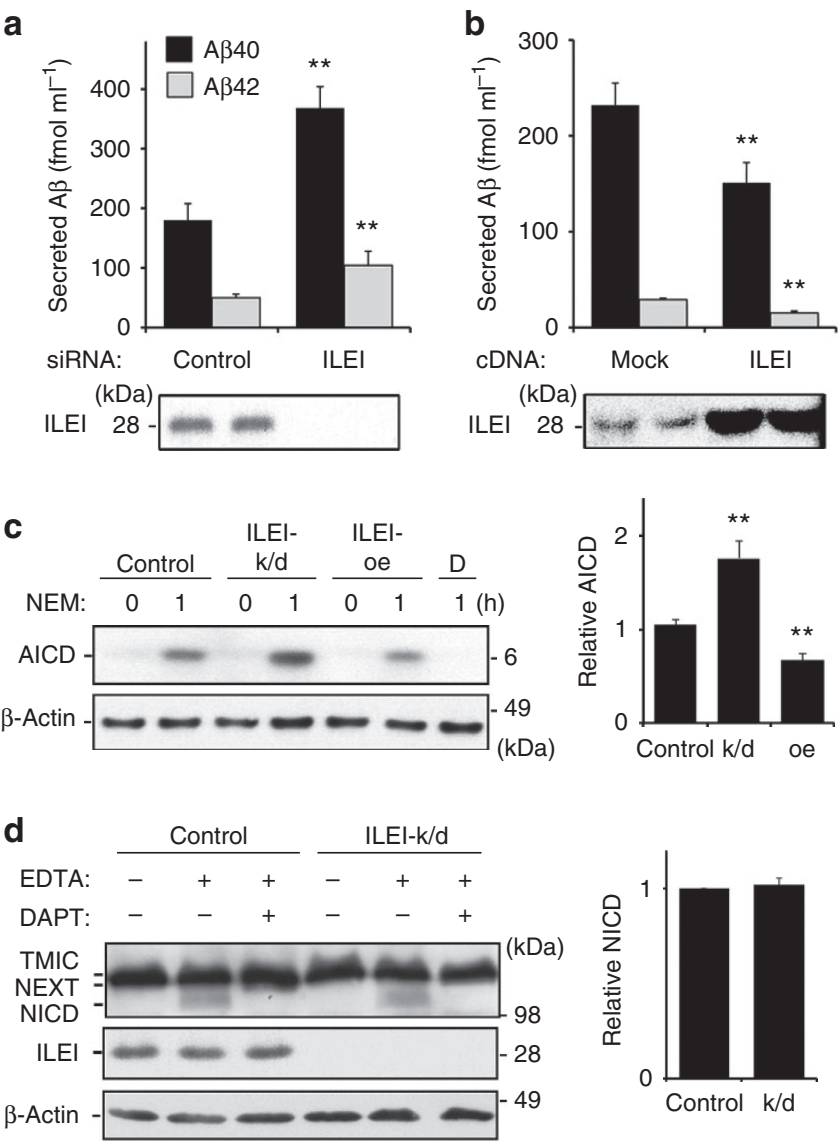

Figure 2 | ILEI inhibits cellular $\gamma$-secretase cleavage of APP but not Notch. Non-targeting control or ILEI-specific siRNA (a), or mock or ILEI cDNA (b) was transiently transfected into HEK293 cells. A 340 (black bars) and $A \beta 42$ (grey bars) in the conditioned medium were measured using ELISA assays ( $n=6$, mean \pm s.d.). ${ }^{\star \star} P<0.01$ versus the control or the mock by Student's t-test. Lower panels show immunoblots of ILEI. (c) Immunoblot for APP intracellular domain (AICD) in control, ILEI-knockdown $(k / d)$ or ILEl-overexpressing (oe) HEK293 cells. Cells were treated with $200 \mu \mathrm{M}$ $\mathrm{N}$-ethylmaleimide (NEM) for $1 \mathrm{~h}$ to block degradation of $\mathrm{AICD}^{60}$. AICD production was inhibited by DAPT ( $N$-[N-(3,5-difluorophenacetyl)---alanyl]$S$-phenylglycine $t$-butyl ester; $1 \mu \mathrm{M})$ treatment $(\mathbf{d})$. The graph shows the relative intensity of AICD normalized to $\beta$-actin ( $n=3$, mean \pm s.d.).

${ }^{\star \star} P<0.01$ versus the control by Student's $t$-test. (d) Proteolysed derivatives of endogenous Notch-1 in control and ILEI-knockdown $(k / d)$ mouse embryonic fibroblasts (MEFs). EDTA $(1.5 \mathrm{mM})$ treatment induced cleavage of transmembrane/intracellular Notch (TMIC) to generate Notch

extracellular truncation (NEXT), which was then cleaved by $\gamma$-secretase to release Notch intracellular domain (NICD). NICD production was inhibited by DAPT $(1 \mu \mathrm{M})$ treatment. The graph shows the relative intensity of NICD normalized to $\beta$-actin $(n=3$, mean \pm s.d.). No significant difference versus the control was found ( $P>0.05$ by Student's $t$-test).

knockdown in mouse embryonic fibroblasts did not alter Notch intracellular domain generation from endogenous Notch in the presence of EDTA (Fig. 2d). In addition, we used a cell-based luciferase reporter assay for $\gamma$-secretase cleavage of Notch. The specificity was confirmed by treatment with a potent $\gamma$-secretase inhibitor, DAPT ( $N$-[N-(3,5-difluorophenacetyl)-L-alanyl]-Sphenylglycine $t$-butyl ester), which attenuated luminescence $(35.9 \pm 4.5 \%$ of vehicle; vehicle, $100.0 \pm 6.5 \% ; n=5, P=0.000)$. Induction of luciferase activity was unaffected by transfection with ILEI siRNA $(94.5 \pm 12.5 \%$ of control; control, $100.0 \pm 5.7 \%$; $n=5, P=0.449)$ or ILEI complementary DNA $(96.8 \pm 8.9 \%$ of mock; mock, $100.0 \pm 8.2 \% ; n=5, P=0.596)$. Thus, ILEI does not perturb $\gamma$-secretase processing of Notch.

To test whether ILEI directly inhibits $\gamma$-secretase activity, we evaluated $A \beta$ generation in a cell-free assay in which microsome fractions derived from HEK293 cells treated with non-targeting control or ILEI-specific siRNA were incubated with excess recombinant APP-CTF $\beta$. Unexpectedly, we found no significant difference in $\mathrm{A} \beta$ generation $(n=6, P=0.851$ for $\mathrm{A} \beta 40 ; P=0.561$ for $\mathrm{A} \beta 42)$ between control (A $\beta 40: 3,253.3 \pm 399.0, \mathrm{~A} \beta 42$ : $597.7 \pm 23.8 \mathrm{fmol} \mathrm{ml}^{-1}$ ) and ILEI-knockdown cells (A $\beta 40$ : $\left.3,181.9 \pm 306.4, A \beta 42: 614.5 \pm 28.9 \mathrm{fmol} \mathrm{ml}^{-1}\right)$, indicating that ILEI reduces cellular $A \beta$ generation without inhibiting $\gamma$-secretase activity. This result and the observation that ILEI was processed normally in the presence of DAPT (Supplementary Fig. 2a) further suggest that ILEI is not a competitive substrate of $\gamma$-secretase. In addition, ILEI knockdown did not detectably affect the cellular expression levels or subcellular localization of $\gamma$-secretase components, the intracellular trafficking of APP, or the $A \beta$ secretion process (Supplementary Figs 2b-d and $3 \mathrm{a}$ ). We also excluded the possibility that ILEI accelerated degradation of secreted $A \beta$ in the culture medium (Supplementary Fig. 3b).

Accordingly, ILEI knockdown increased the level of cellular APP-CTFs (APP-CTF $\alpha$ and APP-CTF $\beta$ ) without augmenting APP-FL or secreted ectodomains of APP (Fig. 3a). ILEI knockdown did not change APP transcript expression as measured with quantitative real-time PCR $(104.1 \pm 3.8 \%$ of control; control, $100.0 \pm 2.9 \% ; n=3, P=0.291)$ or cellular $\beta$-secretase activity as measured with an in vitro assay $(104.4 \pm 7.31 \%$ of control; control, $100.0 \pm 6.1 \% ; n=3, P=0.555)$. We thus reasoned that ILEI knockdown stabilizes APP-CTF $\beta$ to increase A $\beta$ generation. A cycloheximide chase assay indicated that ILEI knockdown significantly extended the half-life of APP-CTFs but not APP-FL (Fig. 3b). Altered ILEI expression levels did not change the levels of Beclin-1 or Rab5a or the subcellular localization of APP-CTFs (Supplementary Fig. 3a and c), suggesting that stabilization of APP-CTFs is not mediated by inactivation of general protein degradation through lysosomal/autophagosomal pathways or a defect in APP-CTF trafficking. The effect of ILEI knockdown on APP metabolism could be rescued by transfection of siRNAresistant ILEI cDNA (Fig. 3c), ruling out potential off-target effects of the siRNA-mediated RNAi.

We examined whether ILEI also acts on low-density lipoprotein receptor-related protein 1 (LRP1) and N-cadherin, which are other substrates of $\gamma$-secretase. Knockdown or overexpression of ILEI did not affect accumulated levels of LRP1 or N-cadherin CTFs (Fig. 3d). Taken together with the results for Notch-1 (Fig. 2d), our results suggest that ILEI exhibits selectivity for APP-CTFs.

ILEI interferes with the APP-CTF-stabilizing property of PS1. Processed ILEI is secreted from the cell, whereas intracellular ILEI is mostly associated with the microsome fraction (Supplementary Fig. 4a). Hence, we next determined whether extracellular application of a secreted form of ILEI decreased cellular $A \beta$ secretion. We purified a recombinant polypeptide of processed ILEI from the conditioned medium of C-terminally V5-His-tagged ILEI (ILEI-VH)-overexpressing HEK293 cells (Supplementary Fig. 4b). When added into the culture medium of ILEIknockdown HEK293 cells, purified ILEI-VH suppressed the levels of secreted $A \beta$ and cellular APP-CTFs in a dose-dependent manner (Fig. 4a). Purified ILEI-VH was detected in perinuclear vesicular compartments and was associated with PS1 (Fig. 4b,c). These results suggest that ILEI acts on the extracellular side of the cell membrane and is endocytosed into the cell. In contrast, when we added purified ILEI-VH to cell-free mixtures and performed 
a

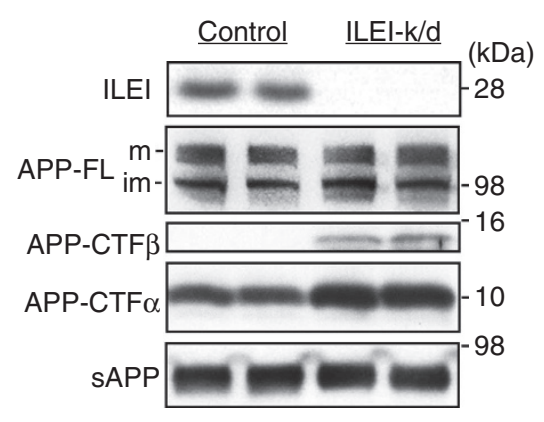

c

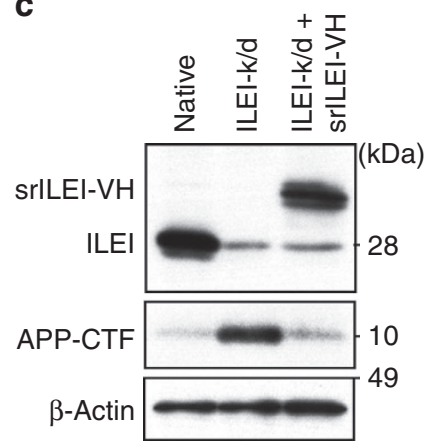

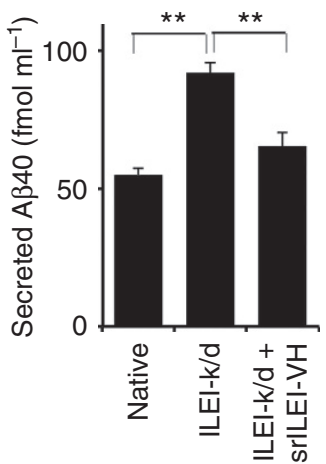

b
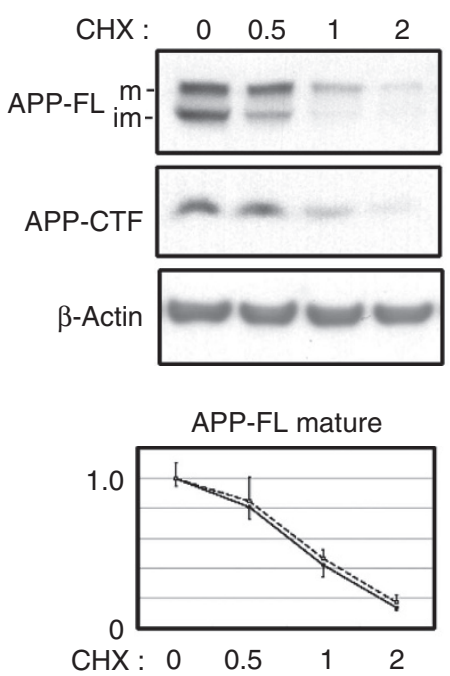

APP-FL immature

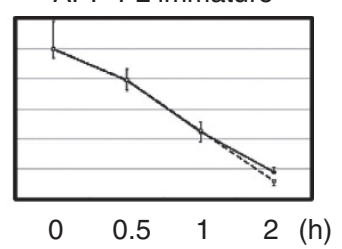

d
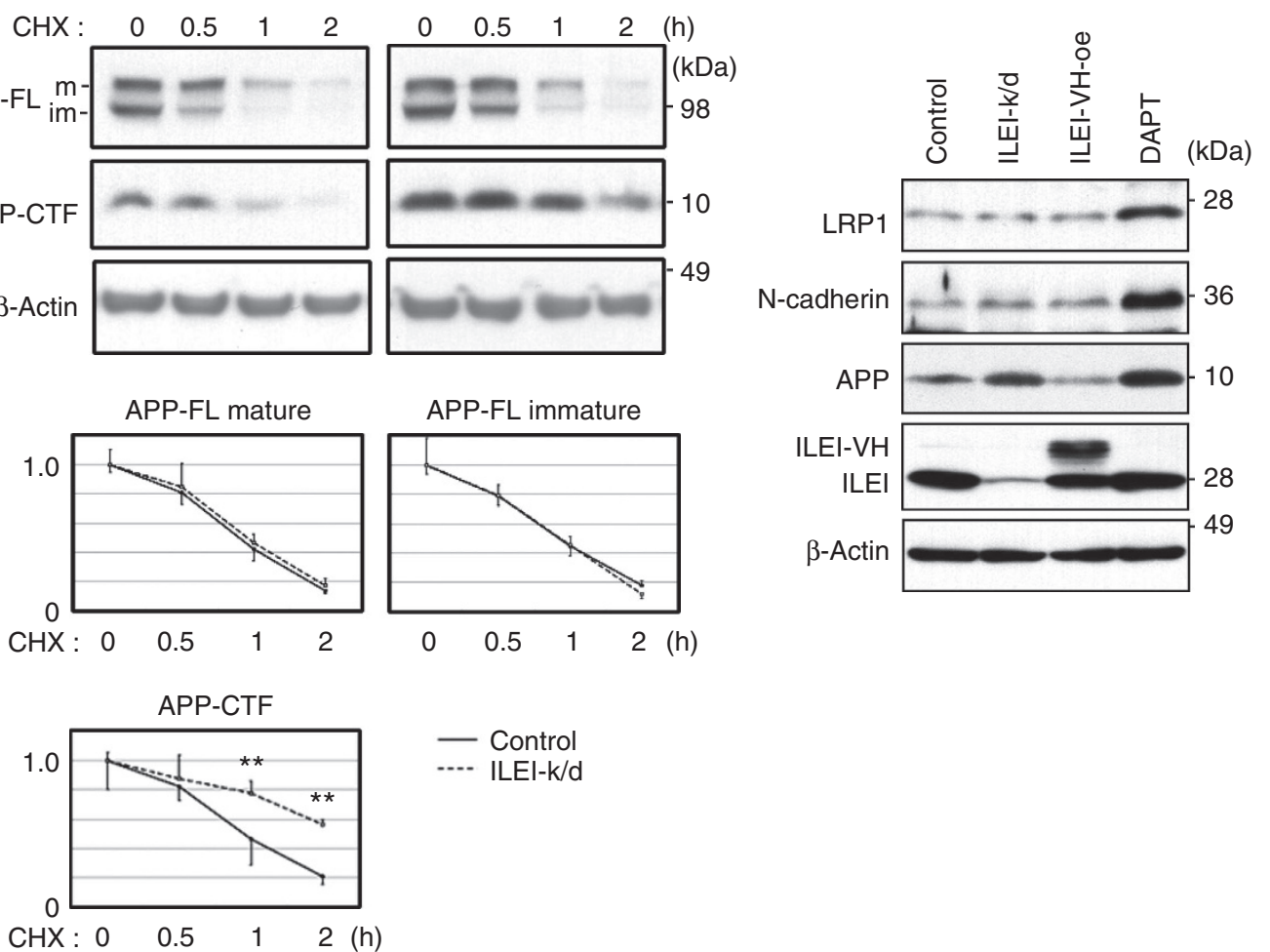

- Control

-..- ILEI-k/d

Figure 3 | ILEI knockdown selectively stabilizes APP-CTFs. (a) Immunoblots for APP and its proteolysed derivatives in control and ILEI-knockdown (k/d) HEK293 cells. The same amount of protein from cell lysates (for ILEI, APP FL and APP-CTFs) or conditioned medium (for secreted ectodomain; sAPP) was loaded. For APP-CTF $\beta$, a longer exposure image is shown. m, mature; im, immature. (b) Immunoblots of APP-FL and APP-CTF in a cycloheximide (CHX) chase assay. m, mature; im, immature. Non-targeting control or ILEI-specific siRNA-transfected HEK293 cells were treated with $50 \mu \mathrm{ml} \mathrm{ml}^{-1} \mathrm{CHX}$ for the indicated times. $\beta$-Actin was used as a loading control. The graphs below show the relative intensity of the bands $\left(n=5\right.$, mean \pm s.d.). ${ }^{\star \star} P<0.01$ versus the control by Student's $t$-test. (c) The restoring effect of siRNA-resistant ILEI-V5-His (srlLEI-VH) on the levels of APP-CTFs and secreted A 3 . ILEI-specific siRNA-transfected HEK293 cells were further transfected with srlLEI-VH. APP-CTF was analysed with immunoblotting, and secreted A 340 was measured with ELISA ( $n=3$, mean \pm s.d.). ${ }^{* \star} P<0.01$ by Student's $t$-test. (d) Immunoblots for LRP1-CTF $(25 \mathrm{kDa}), \mathrm{N}$-cadherin-CTF (36 kDa) and APP-CTF (10 kDa) in control, stable ILEI-knockdown (k/d), stable ILEI-VH-overexpressing (oe) and DAPT ( $N$-[N-(3,5-difluorophenacetyl)-L-alanyl]-S-phenylglycine t-butyl ester; $1 \mu \mathrm{M}$ )-treated HEK293 cells. $\beta$-Actin was used as a loading control.

$\gamma$-secretase assays using microsome fractions derived from ILEIknockdown HEK293 cells, A $\beta$ generation was not altered (Supplementary Fig. 4c).

Although we identified ILEI as a $\gamma$-secretase complex-binding protein, whether ILEI interaction with the $\gamma$-secretase complex mediated the stabilization of APP-CTFs was not clear. To address this issue, we tested the effect of ILEI knockdown on APP-CTF levels in PS1/PS2-knockdown HEK293 cells. High levels of APPCTFs accumulated in PS1/PS2-knockdown cells in which the $\gamma$-secretase complex was ablated. ILEI knockdown did not lead to any further increase in APP-CTFs (Fig. 4d). In contrast, as shown in Fig. 4d, the APP-CTF level was increased by ILEI knockdown in PEN-2-knockdown HEK293 cells, which exclusively contained immature, inactive $\gamma$-secretase complexes ${ }^{24}$. Furthermore, ILEI knockdown and ILEI-VH treatment enhanced and reduced accumulation of APP-CTFs in the presence of a $\gamma$-secretase inhibitor, respectively (Fig. 4a,d). Thus, ILEI requires interaction with the $\gamma$-secretase complex, regardless of whether the complex is enzymatically active or inactive, to alter the stability of APP-CTFs. In contrast, co-immunoprecipitation assays revealed no direct interaction between ILEI and APP-CTFs (Supplementary Fig. 3d).

We next asked whether ILEI competes with APP-CTFs for binding with the $\gamma$-secretase complex. Co-immunoprecipitation 
assays indicated that the amount of PS1-bound APP-CTFs was inversely correlated with the ILEI expression level (Fig. 4e). In contrast, alternative co-immunoprecipitation assays suggested that the expression level of APP did not influence the amount of PS1-bound ILEI (Fig. 4f). These results imply that ILEI non-competitively interferes with the binding between the $\gamma$-secretase complex and APP-CTFs.

The PS $1 / \gamma$-secretase complex stably binds APP-CTFs and protects them from non-specific degradation ${ }^{21,25}$. Analysis of the APP-CTF-stabilizing property is hampered by $\gamma$-secretase

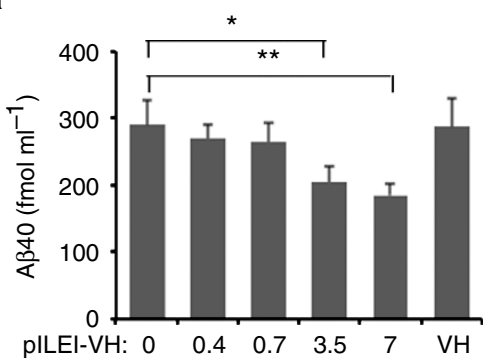

b

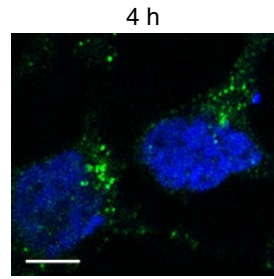

$\mathrm{Oh}$

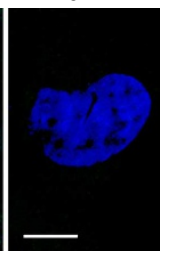

PILEI-VH : $\quad 0 \quad 3.5 \quad 7 \quad 0 \quad 7$
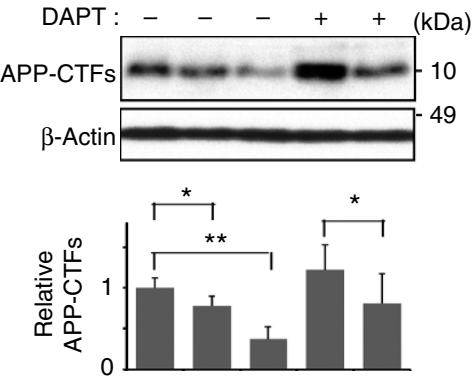

C

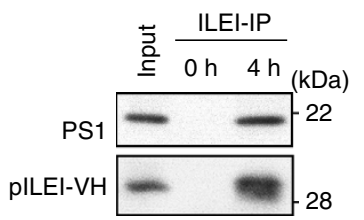

d
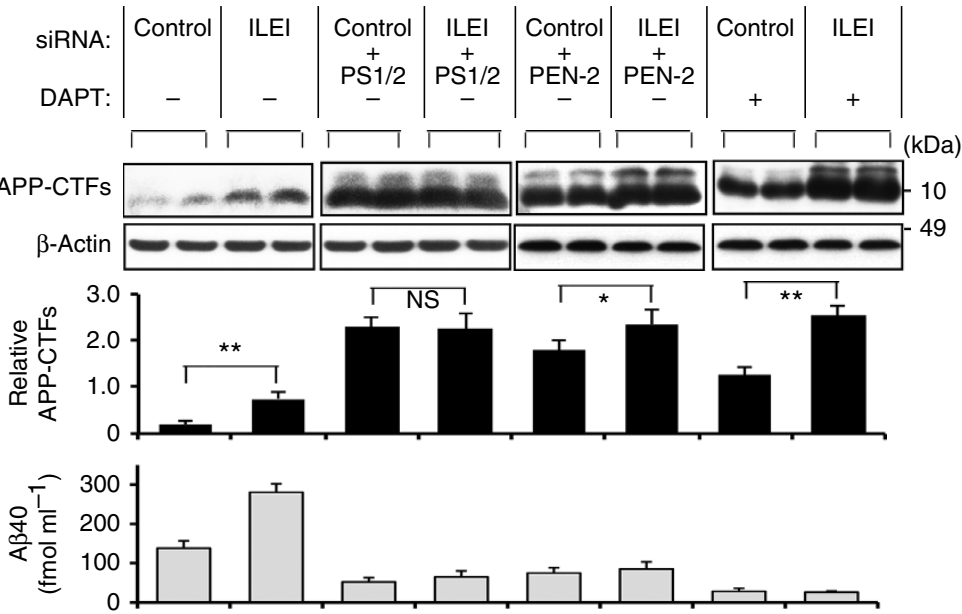

e

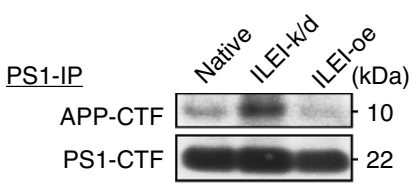

Cell lysate

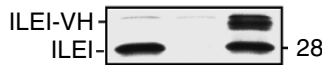

$\mathbf{f}$

PS1-IP

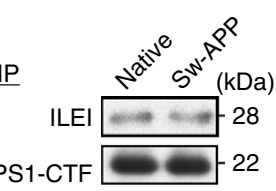

Cell lysate

APP-CTF

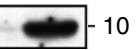

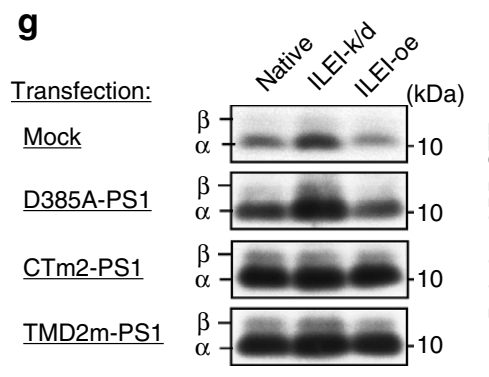

Mock $\square$ CTm2-PS1

D385A-PS1 $\mathbb{N}$ TMD2m-PS1

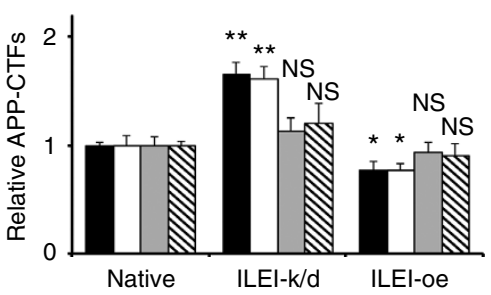


activity, although the former is independent of the latter ${ }^{25}$. To determine whether ILEI interferes with this property, we used three catalytically inactive PS1 mutants: D385A-PS1, CTm2-PS1 and TMD2m-PS1. D385A-PS1 has an alanine substitution of the aspartic acid residue $\left(\mathrm{D}^{385}\right)$ at the active centre and binds with APP-CTFs and ILEI (Supplementary Fig. 5a-c). CTm2-PS1 has alanine substitutions of $\mathrm{D}^{385}$ and the sequential three amino acid residues $\left(\mathrm{D}^{458}, \mathrm{Q}^{459}\right.$ and $\left.\mathrm{L}^{460}\right)$ in the extracellular/luminal C-terminal tail and lacks the ability to bind ILEI (Supplementary Fig. 5a,b). TMD2m-PS1, the second transmembrane domain (TMD) of which is replaced with the TMD of CD4 (ref. 26), lacks the ability to bind APP-CTFs (Supplementary Fig. 5c). Overexpression of D385A-PS1 or CTm2-PS1 but not TMD2m-PS1 increased APP-CTFs in PS1/PS2-double knockout mouse embryonic fibroblasts ${ }^{27}$ (Supplementary Fig. 5d), suggesting that APP-CTFs are stabilized by the binding with PS1. The APP-CTF-destabilizing effect of ILEI was abrogated by overexpression of CTm2-PS1 or TMD2m-PS1 (Fig. 4g), suggesting that both mutants prevented ILEI from destabilizing APP-CTFs in a dominant-negative manner. Exogenous CTm2PS1 and TMD2m-PS1 are expected to compete with endogenous PS1 for binding to APP-CTFs and ILEI, respectively. Our results thus imply that APP-CTF destabilization by ILEI requires the interaction between ILEI and PS1, and between PS1 and APP-CTFs. APP-CTFs that accumulated with ILEI knockdown were indeed colocalized with PS1 in subcellular fractions of cultured cells (Supplementary Fig. 3a). These results support our hypothesis that ILEI binds to the PS $1 / \gamma$-secretase complex and interferes with its APP-CTF-stabilizing property to reduce A $\beta$ generation.

Neuronal expression of ILEI. Previous studies showed that ILEI is expressed in the central nervous system as well as in secretory epithelia ${ }^{22,28}$. However, the regional and cellular distribution of ILEI in mammalian brains has not been examined. We confirmed the specificity of rabbit anti-ILEI antibody (Supplementary Fig. 6) and performed immunohistochemical staining for ILEI. ILEI was widely expressed in mouse and human brains, and was prominent in pyramidal neurons in the cerebral cortex and hippocampus. ILEI-immunoreactive structures were detected in perinuclear regions of neuronal cell bodies, whereas glial fibrillary acidic protein-positive astrocytes and Iba1-positive microglia were negative for ILEI (Fig. 5a). Perinuclear ILEI was colocalized with TGN46, Rab5a and Rab7, indicating that ILEI principally resides in the trans-Golgi network and the endocytic vesicles where $A \beta$ is reportedly produced ${ }^{29,30}$ (Supplementary Fig. 7).
Colocalization of ILEI and APP-CTFs was also confirmed (Supplementary Fig. 8).

Recently, transforming growth factor- $\beta$ (TGF- $\beta$ ) signalling in neuronal cells was reported to lead to decreased levels of APPCTF and $A \beta^{31}$. On the other hand, TGF- $\beta$ selectively induces translation of ILEI via phosphorylation of heterogeneous nuclear ribonucleoprotein E1 in mammary gland cells ${ }^{32}$. Thus, we asked whether the inhibitory effect of TGF- $\beta$ on $A \beta$ production in brain is mediated by ILEI induction. To address this, we first examined ILEI induction and $A \beta$ reduction by TGF- $\beta$ using organotypic culture of rat forebrain slices. Next, we evaluated TGF- $\beta$-induced reduction in $A \beta$ production from forebrain slices pretreated with ILEI-specific siRNA. TGF- $\beta$ induced ILEI and reduced $A \beta$ secretion, and these effects were almost completely abrogated in the ILEI-knockdown condition (Fig. 5b).

To evaluate the possible involvement of ILEI in AD pathogenesis, we examined the levels of secreted ILEI in autopsy brains of patients with sporadic AD. Soluble fractions from temporal cortex homogenates were subjected to immunoblotting. ILEI expression was normalized to the level of neuron-specific enolase in each sample to compensate for the difference in neuronal density. ILEI was significantly reduced in $\mathrm{AD}$ cases compared with age-matched non-neurological disease controls (Fig. 5c, $P=0.000)$ and was inversely correlated with levels of $\mathrm{A} \beta$ or phosphorylated tau with semi-quantitative immunoblotting (Supplementary Fig. 9a,b). The ILEI level in autopsy brains with non-AD neurological diseases was equivalent to that of nonneurological disease control brains (Fig. 5c, $P=0.459$ ). Although our result is preliminary due to the limited number of cases examined, the trend is unequivocal. The decrease in ILEI did not seem to be secondary to brain A $\beta$ accumulation, because ILEI is unaltered in the brains of aged APP-overexpressing mice ${ }^{33}$ (Supplementary Fig. 9c).

ILEI ameliorates the phenotypes of AD model mice. To analyse the consequences of ILEI overexpression in mammalian brains, we developed $\mathrm{Tg}$ mice in which the mouse prion promoter was used to drive expression of a human ILEI cDNA transgene predominantly in the brain ${ }^{34}$. Heterozygous $\mathrm{Tg}$ mice exhibited normal development and fertility with no gross morphological defects. Similar to wild-type mice, ILEI expression was restricted to neuronal cells in the brains of $\mathrm{Tg}$ mice (Fig. 6a). Immunoblotting of brain homogenates indicated a three-fold increase in ILEI protein levels and no alteration in Notch intracellular domain levels in the Tg mice compared with non- $\mathrm{Tg}$ littermates (Fig. 6b). These findings suggest that the ILEI-Tg mice

Figure 4 | Secreted ILEI interacts with the PS1/ $\gamma$-secretase complex and interferes with its APP-CTF-stabilizing property. (a) Effects of purified ILEIVH (pILEI-VH) on A $\beta$ secretion and cellular APP-CTFs. pILEI-VH was added to the culture medium of ILEI-knockdown HEK293 cells at the indicated concentrations $\left(\mu \mathrm{g} \mathrm{ml}^{-1}\right)$. V5-His tag peptide $\left(\mathrm{VH}, 7 \mu \mathrm{g} \mathrm{ml}^{-1}\right)$ served as a negative control. Secreted A 340 was measured with ELISA assay and APP-CTFs were analysed with immunoblotting. The right graph shows the relative intensity of APP-CTFs normalized to $\beta$-actin ( $n=3$, mean \pm s.d.). (b) Laser scanning confocal microscopic images of pILEI-VH. pILEI-VH was immunostained with anti-V5 tag antibody (green) 0 or $4 \mathrm{~h}$ after its addition to the medium. Nuclei were stained with Hoechst 33342 (blue). Scale bar, $10 \mu \mathrm{m}$. (c) Co-immunoprecipitation assays for pILEI-VH and PS1. ILEI-knockdown HEK293 cells were lysed 0 or $4 \mathrm{~h}$ after addition of pILEI- $\mathrm{VH}$, and the cell lysates were subjected to immunoprecipitation. A blot of the anti-ILEl precipitate was probed with anti-PS1-CTF or anti-V5 antibody. (d) ILEl-knockdown effects on APP-CTFs and secreted A 340 levels in PS1/PS2-knockdown, PEN-2-knockdown, or DAPT ( $N$-[N-(3,5-difluorophenacetyl)-L-alanyl]-S-phenylglycine $t$-butyl ester; $1 \mu \mathrm{M})$-treated HEK293 cells $(n=3$, mean \pm s.d.). (e) Co-immunoprecipitation assays for PS1 and APP-CTFs. Cell lysates of native, ILEI-knockdown (k/d) and ILEl-overexpressing (oe) HEK293 cells were immunoprecipitated with antiPS1 antibody (PS1-IP). (f) Co-immunoprecipitation assays for PS1 and ILEI. Cell lysates of native and Swedish APP-overexpressing (Sw-APP) HEK293 cells were immunoprecipitated with anti-PS1 antibody (PS1-IP). (g) Effects of PS1 mutants on ILEl function. Mock, D385A-PS1, CTm2-PS1 or TMD2m-PS1 was transfected into native, ILEl-knockdown (k/d) and ILEl-overexpressing (oe) HEK293 cells (the same cell lines as in e). The same amount of protein from cell membrane lysates was subjected to immunoblotting for APP-CTFs. $\alpha$ : APP-CTF $\alpha, \beta$ : APP-CTF $\beta$. The graph shows the relative intensity of APP-CTFs normalized to native HEK293 cells transfected with the same plasmid ( $n=3$, mean \pm s.d.). For data from $\mathbf{a}, \mathbf{d}$ and $\mathbf{g}$, two-tailed Student's $t$-test was used to analyse statistical significance. ${ }^{\star} P<0.05,{ }^{\star \star} P<0.01$ and NS, not significant, versus control $(\mathbf{a}, \mathbf{d})$ or native $(\mathbf{g})$. 
a ILEI

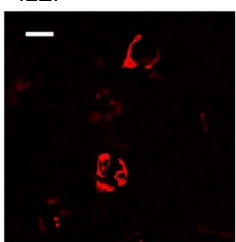

ILEI + GFAP

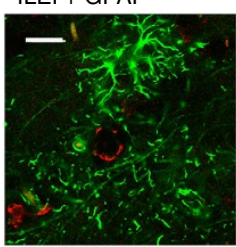

b

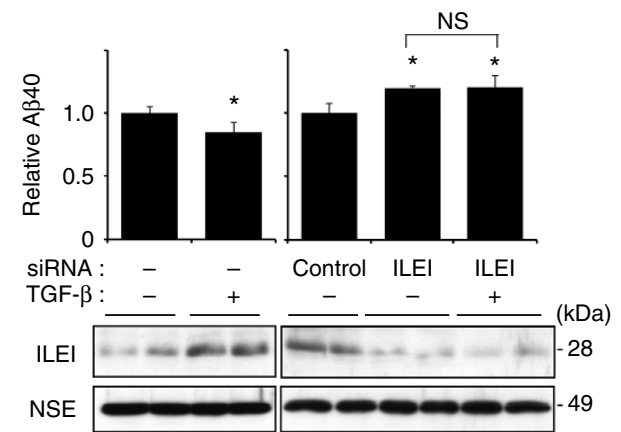

C

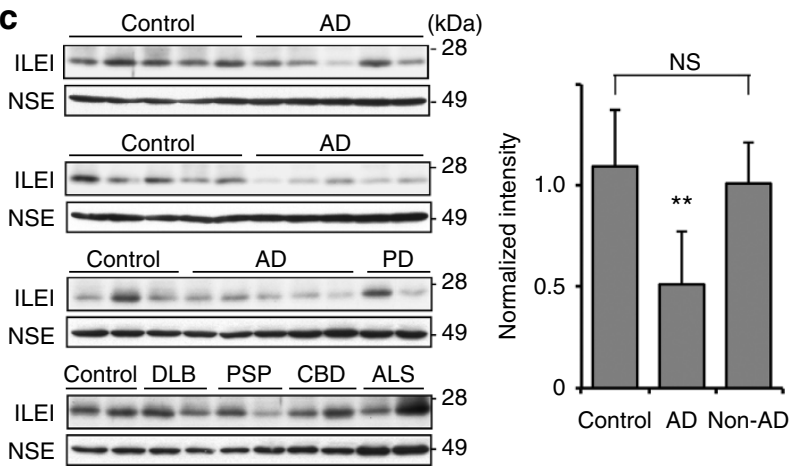

Figure 5 | Neuronal expression of ILEI is induced by TGF- $\beta$ and reduced in AD brains. (a) Double immunostaining for ILEI and cell type-specific markers in mouse brain sections. Neurofilament (NF), glial fibrillary acidic protein (GFAP) and Iba-I are marker proteins for neurons, astrocytes and microglia, respectively. Scale bars, $10 \mu \mathrm{m}$. (b) Effects of TGF- $\beta$ treatment on cultured rat brain slices pretreated with non-targeting control or ILEI-specific siRNA. Forebrain slices containing the hippocampus and cerebral cortex were prepared from three Wistar rats (3-week-old, female). The relative levels of A $\beta 40(n=3$, mean \pm s.d.) and immunoblotting for ILEl are shown. Neuronspecific enolase (NSE) was used as a loading control. ${ }^{\star} P<0.05$ versus vehicle or control, and NS, not significant by Student's $t$-test. $A \beta 40$ concentrations were as follows: control for TGF- $\beta, 743.68 \pm 39.32$; TGF- $\beta, 631.23 \pm 54.36$; control for ILEI-knockdown, 751.77 \pm 60.23 ; ILEI-knockdown, $901.28 \pm 12.61$; ILEI-knockdown/TGF- $\beta, 902.28 \pm 72.57$ pmol per $g$ protein $(n=3$, mean \pm s.d.). (c) Immunoblots for ILEI in Tris-buffered saline-extracted fractions from temporal cortices of AD patients ( $n=15,82.0 \pm 4.5$ years old, post-mortem interval $7.0 \pm 3.5 \mathrm{~h}$ ), age-matched controls without neurological disease $(n=15$, $80.1 \pm 1.7$ years old, post-mortem interval $8.0 \pm 5.4 \mathrm{~h}$ ) and non-AD neurological disease controls ( $n=10,79.6 \pm 3.7$ years old, post-mortem interval 9.3 $\pm 5.9 \mathrm{~h}$ ). Non-AD disease controls were Parkinson's disease (PD), dementia with Lewy bodies (DLB), progressive supranuclear palsy (PSP), corticobasal degeneration (CBD) or amyotrophic lateral sclerosis (ALS). The graph shows the relative intensity of ILEl normalized to NSE (mean \pm s.d.). ${ }^{\star} P<0.01$ versus control or non-AD control and NS, not significant by Student's $t$-test. represent an appropriate model for ILEI overexpression without obvious ectopic expression. Immunoblotting revealed a $30 \%$ decrease in APP-CTF $\alpha$ and APP-CTF $\beta$ levels in the brains of Tg mice compared with controls (Fig. 6c), and the endogenous levels of $A \beta 40$ and $A \beta 42$ in Tris-buffered saline-soluble and -insoluble fractions of brain homogenates were significantly reduced in ILEI-Tg mice compared with non-Tg littermate controls (Fig. 6d). Thus, overexpression of ILEI suppresses APP-CTF accumulation and $\mathrm{A} \beta$ generation in vivo while sparing Notch processing.

To determine whether ILEI overexpression affects disease progression, we bred ILEI-Tg mice with Swedish mutant APPoverexpressing mice (APP-Tg, Tg2576), which show impaired learning and memory and brain $A \beta$ deposition by $9-10$ months of age ${ }^{33}$. APP/ILEI-double Tg mice appeared healthy and had a survival curve comparable with APP-Tg mice. We used a Y-maze test to assess hippocampus-dependent spatial working memory, which declines with age in APP-Tg mice ${ }^{33}$. At 11-13 months of age, but not at 6 months of age, APP-Tg mice showed poor performance $(n=12, P=0.0000)$, but APP/ILEI-Tg mice showed similar performance $(n=11, P=0.3692)$ compared with non-Tg littermate (Fig. 7a). We found no significant difference in locomotor activity or motivation to explore the maze (the total number of arm entries was 28.6 \pm 3.75 in APP-Tg mice and $25.2 \pm 4.08$ in APP/ILEI-Tg mice; $P=0.5478$ ).

We next quantified the $A \beta$ plaque load using brain sections immunohistochemically stained for $A \beta$. At 12 months of age, the plaque number and the area occupied by plaques in the cerebral cortex and hippocampus were clearly reduced in APP/ILEI-Tg mice compared with APP-Tg littermates (Fig. 7b-d). Moreover, quantitative analysis with $\mathrm{A} \beta$ enzyme-linked immunosorbent assay (ELISA) using brains of the same set of mice confirmed that the levels of $A \beta 40$ and $A \beta 42$ in soluble and insoluble fractions were significantly lower in APP/ILEI-Tg mice than in APP-Tg littermates (Fig. 7e,f). These results support the conclusion that ILEI efficiently reduces $A \beta$ accumulation and ameliorates the memory deficit caused by $\mathrm{A} \beta$ overload in the brain.

\section{Discussion}

Our study shows that $\mathrm{A} \beta$ generation can be attenuated by overexpression or administration of ILEI. ILEI regulated $A \beta$ generation by altering the stability of APP-CTFs by extracellular or luminal binding to the PS $1 / \gamma$-secretase complex. ILEI exhibited functional selectivity for APP and did not affect Notch derivatives. Neuronal expression of ILEI was enhanced by TGF$\beta$ signalling in mammalian brain. The levels of secreted ILEI were reduced in autopsy brains of patients with sporadic AD. Tg overexpression of ILEI successfully ameliorated brain A $\beta$ burden and memory deficits in AD model mice.

Based on a recent structural study showing that ILEI and pancreatic-derived factor (PANDER, also known as FAM3B) adopt a globular $\beta \beta \alpha$ fold that is distinct from the conformation of classical cytokines, the FAM3 superfamily members may represent a novel class of signalling molecules ${ }^{35}$. Earlier works have revealed that ILEI acts downstream of TGF- $\beta$ to induce epithelial-to-mesenchymal transition of epithelial cells, whereas PANDER, which is cosecreted with insulin from pancreatic islet cells, is involved in regulation of insulin secretion and glycemic levels ${ }^{28,36,37}$. Expression of the ILEI transcript in human brain has been described ${ }^{22}$. However, the function of ILEI in the nervous system remains largely unknown, except for its possible role in development of the Xenopus retina ${ }^{38}$. Our results suggest that ILEI is induced by TGF- $\beta$ also in mammalian brain. Multiple lines of evidence link TGF- $\beta$ signalling to brain $A \beta$ accumulation and development of $\mathrm{AD}^{39}$. Thus, the specific genotype $+10 \mathrm{C} / \mathrm{C}$ of the TGF- $\beta 1$ gene, which affects the expression level of TGF- $\beta 1$, 
a
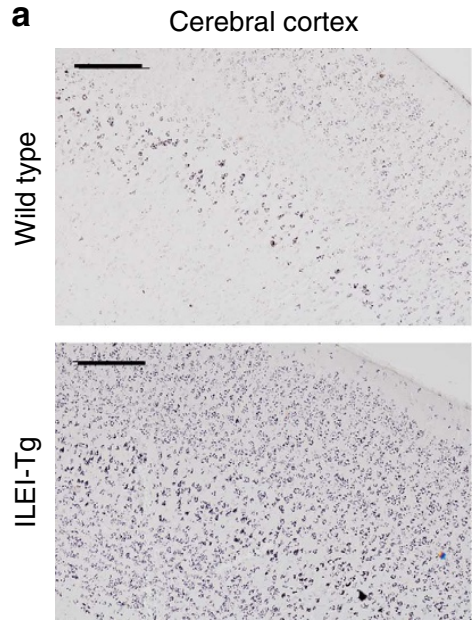

b

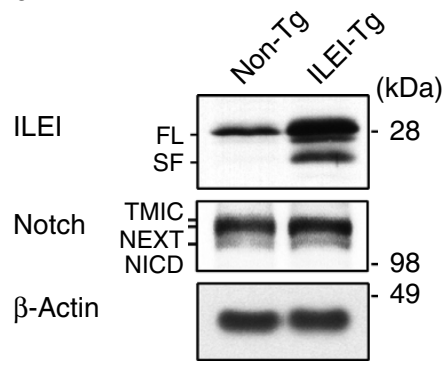

Hippocampus
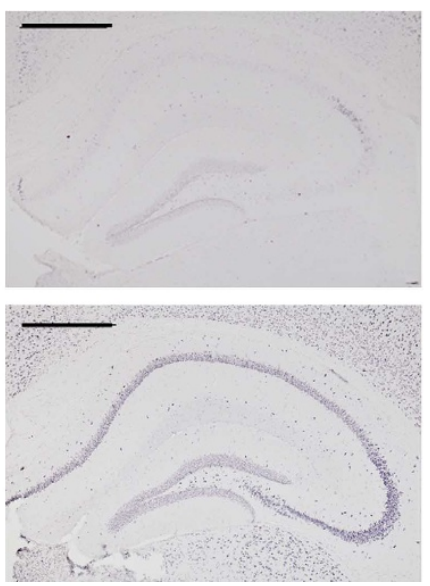

Pyramidal neurons

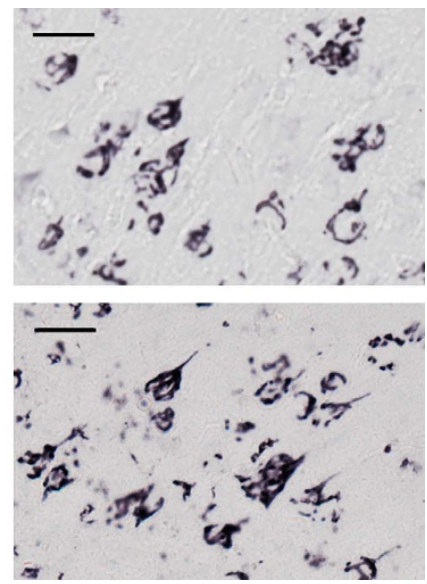

C
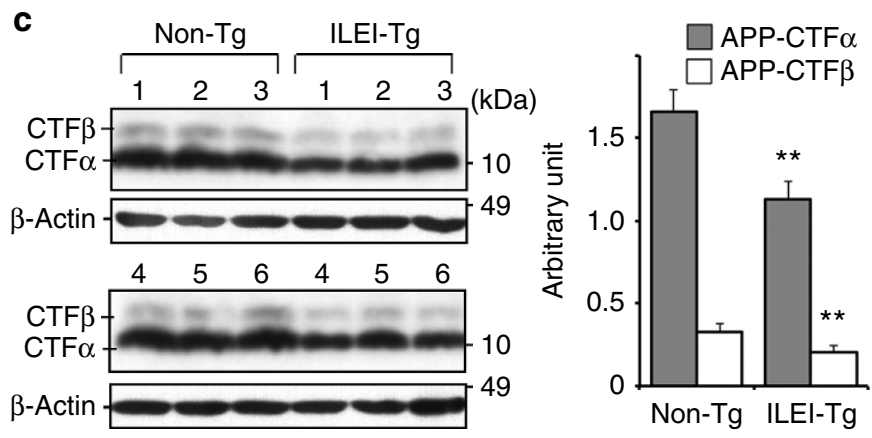

d
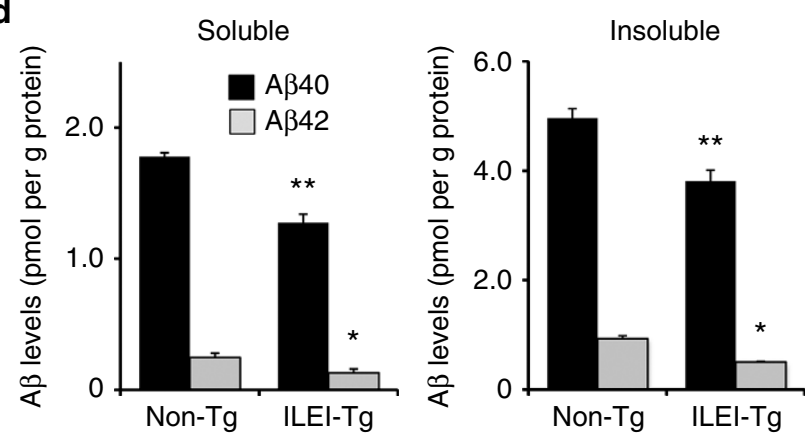

Figure 6 | The A $\boldsymbol{\beta}$ level is reduced in brains of ILEI-Tg mice. (a) Immunostaining shows ILEl expression in neurons of the cerebral cortex and hippocampus of wild-type and ILEI-Tg mouse brains ( 8 months old). Scale bars, $200 \mu \mathrm{m}$ (left), $500 \mu \mathrm{m}$ (centre), $20 \mu \mathrm{m}$ (right). (b) Immunoblots for ILEl and Notch in brains of ILEI-Tg mice and non-Tg littermates ( 6 months old). $\beta$-Actin was used as a loading control. FL, full-length; SF, secreted form. (c) Immunoblots for APP-CTFs in brains of ILEI-Tg mice and non-Tg littermates (6-8 months old, $n=6$ per genotype). The graph shows the relative intensity of APP-CTFs normalized to $\beta$-actin $\left(n=6\right.$, mean \pm s.d.). ${ }^{\star \star} P<0.01$ versus non-Tg mice by Student's t-test. (d) Brain A $\beta$ levels in non- Tg and ILEI-Tg mice (10 months old, $n=6$ per genotype). A $\beta 40$ (black bars) and $A \beta 42$ (grey bars) in Tris-buffered saline-soluble and -insoluble fractions of brain homogenates were measured using ELISA assays $\left(n=6\right.$, mean \pm s.d.). ${ }^{\star} P<0.05$ and ${ }^{\star}{ }^{\star} P<0.01$ versus non-Tg by Student's $t$-test.

is associated with an increased risk of $\mathrm{AD}^{40}$, and the neuronal expression of TGF- $\beta$ type II receptors is reduced in AD brains from the early stage of disease development ${ }^{31}$. In addition, recent proteomic analysis of cerebrospinal fluid revealed significantly decreased ILEI levels in patients with idiopathic temporal lobe epilepsy $^{41}$, in whom the age-related incidence of $A \beta$ plaques in temporal cortex is significantly higher compared with agematched controls ${ }^{42}$. The ILEI level in cerebrospinal fluid may also be a biomarker for $\mathrm{AD}^{43}$. These reports indirectly support our conclusion, indicating the functional relevance of ILEI to A $\beta$ generation in vivo and the pathogenesis of $\mathrm{AD}$.

A previous study using cultured neurons derived from APPCTF $\beta$-Tg mice indicated that $30 \%$ of APP-CTF $\beta$ is converted to
$\mathrm{A} \beta^{44}$. APP-CTFs are also degraded through alternative pathways, including proteasomal, lysosomal and autophagosomal-lysosomal pathways ${ }^{4-47}$. In cultured neuronal cells, proteasome inhibition increases $A \beta$ generation by augmenting the amount of APP$\mathrm{CTF} \beta^{44,45}$. Hence, ILEI knockdown may increase A $\beta$ generation by inhibiting APP-CTF $\beta$ degradation through pathways that do not lead to $A \beta$ production. Alteration of APP-CTF stability by ILEI required cellular expression of the $\gamma$-secretase complex, regardless of whether the complex was enzymatically active or inactive. A large pool of the PS1/ $\gamma$-secretase complexes remains catalytically inactive in the cell ${ }^{48}$. Even active $\gamma$-secretase complexes stably bind to APP-CTFs on substrate-docking sites, which are spatially separated from the catalytic centre ${ }^{21,49}$. 
a

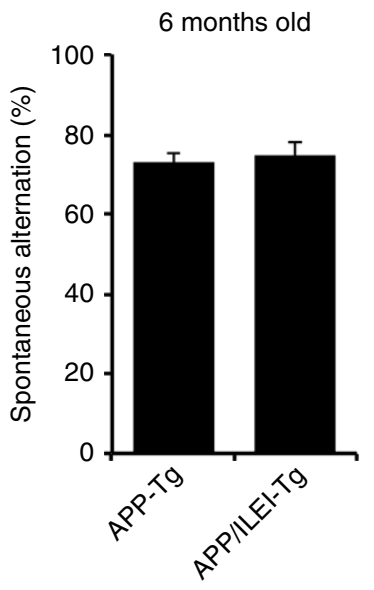

b

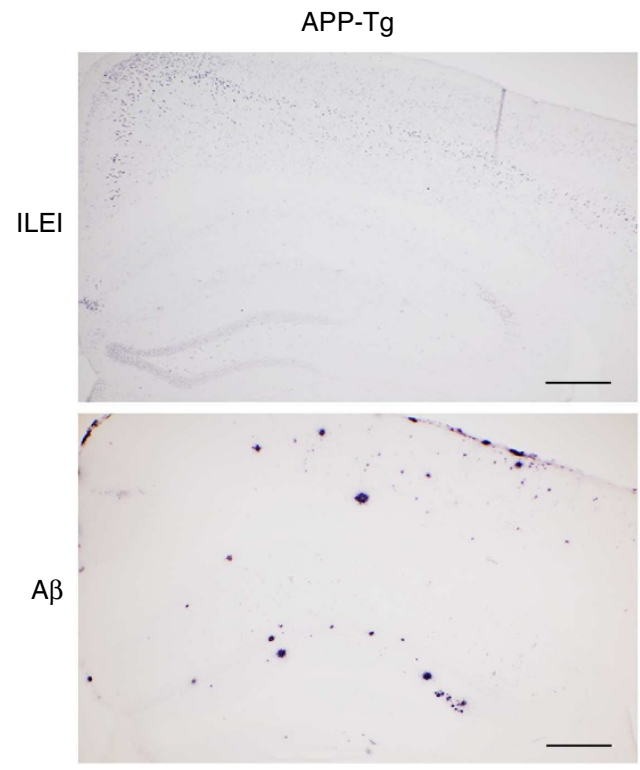

c

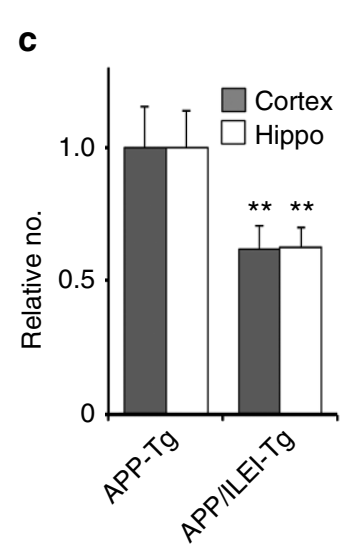

d

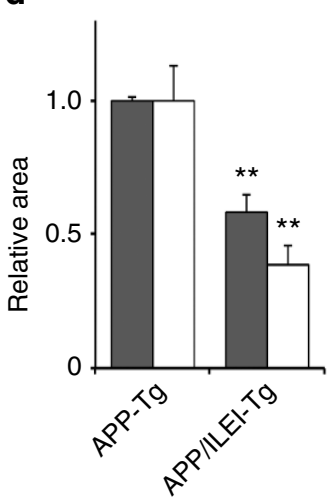

12 months old

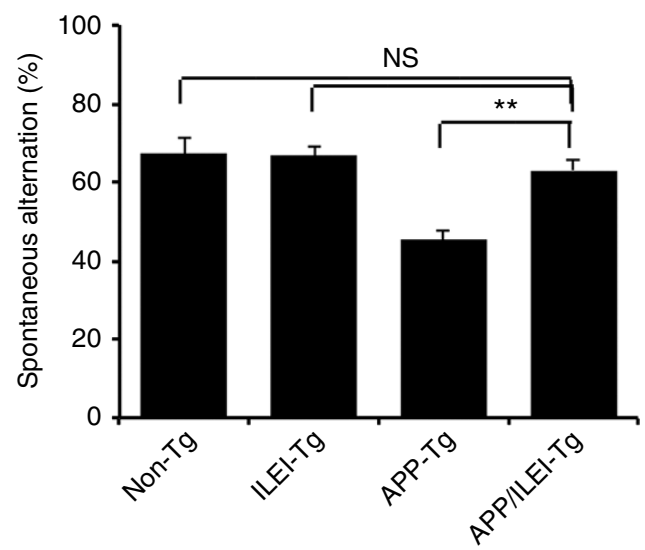

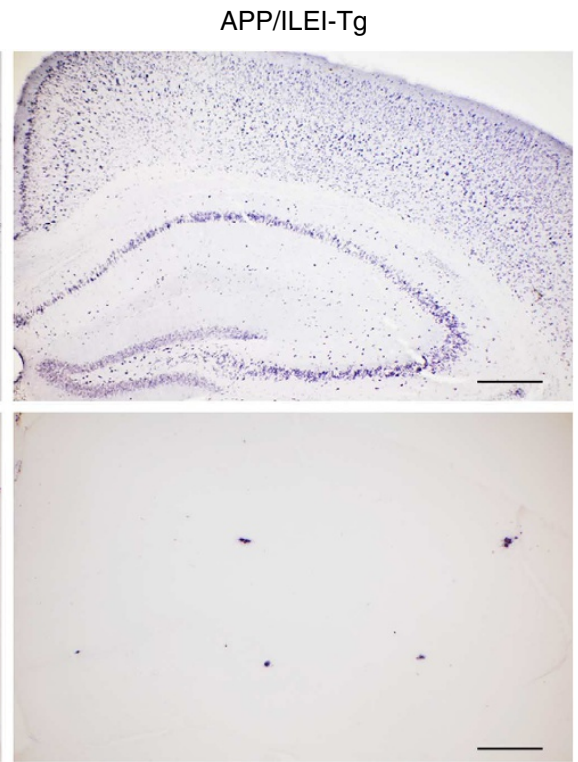
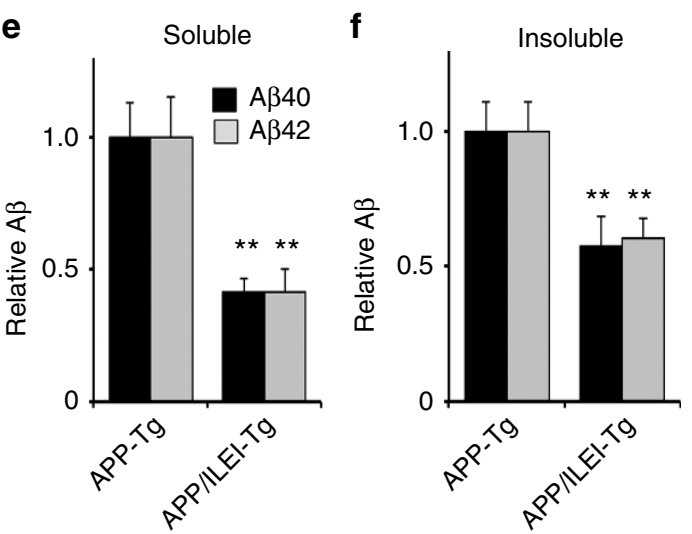

Figure 7 | ILEI overexpression ameliorates the memory deficit and reduces brain A $\beta$ burden in APP-Tg mice. (a) Spontaneous alternation in the Y-maze test. APP-Tg ( 3 females at 6 months age, 6 males and 6 females at 12 months age), ILEI-Tg ( 5 males and 4 females at 12 months age), APP/ILEI-Tg ( 3 females at 6 months age, 5 males and 6 females at 12 months age) and non-Tg ( 5 males and 4 females at 12 months age) littermate mice were used. Data are expressed as percentage (mean \pm s.e.m.). ${ }^{\star \star} P<0.01$, NS, not significant by Student's $t$-test. (b) Immunostaining images with anti-ILEI antibody (top) and anti-A $\beta$ antibody (bottom) using serial brain sections from 12-month-old APP-Tg (left) and APP/ILEI-Tg (right) mice. Scale bars, $200 \mu \mu$. (c-f) A $\beta$ burden in brains of APP-Tg and APP/ILEl-Tg mice (12 months old, $n=6$ per genotype). The plaque number (c) and the area occupied by plaques (d) in $A \beta$-immunostained sections of prefrontal cortex (grey bars) and hippocampus (white bars) (mean \pm s.e.m.). The relative levels of $A \beta 40$ (black bars) and $A \beta 42$ (grey bars) in soluble (e) and insoluble (f) fractions (mean \pm s.e.m.). ${ }^{\star \star} P<0.01$ versus the APP-Tg by Student's $t$-test. A $\beta$ concentrations were as follows: soluble $A \beta 40$ of APP-Tg, 19.79 \pm 2.66 ; soluble $A \beta 42$ of APP-Tg, 4.69 \pm 0.72 ; insoluble A $\beta 40$ of APP-Tg, $1362.56 \pm 152.88$; insoluble $\mathrm{A} \beta 42$ of $\mathrm{APP}-\mathrm{Tg}, 517.80 \pm 57.82$; soluble $\mathrm{A} \beta 40$ of $\mathrm{APP} / \mathrm{ILEl}-\mathrm{Tg}$, $8.14 \pm 1.21$; soluble $\mathrm{A} \beta 42$ of APP/ILEl-Tg, $1.95 \pm 0.42$; insoluble A $\beta 40$ of $\mathrm{APP} / \mathrm{ILEl}-\mathrm{Tg}$, $788.68 \pm 142.23$; insoluble $A \beta 42$ of $\mathrm{APP} / \mathrm{ILEI}-\mathrm{Tg}, 312.92 \pm 38.80$ pmol per g protein (mean \pm s.e.m.). 
Enhanced expression of PS1 in cultured insect and mammalian cells increases the accumulated level of APP-CTF $\beta$ by extending its half-life independent of $\gamma$-secretase activity ${ }^{25}$. Thus, the PS $1 / \gamma$ secretase complex also functions as a chaperone that protects APP-CTFs from nonspecific degradation. Chaperone properties of the $\gamma$-secretase complex have been described only for APP but not for other substrates. Our results indicate that ILEI inhibits this stabilizing ability by directly binding to PS1-CTF without inhibiting $\gamma$-secretase activity, resulting in decreased accumulation of APP-CTFs and diminished generation of A . This possibility is supported by the finding that ILEI is localized in the trans-Golgi network and the endocytic vesicles where PS1:CTF $\beta$ complexes are located and A $\beta$ is produced ${ }^{29,30}$.

Several proteins regulate the stability of APP by directly binding or phosphorylating its cytoplasmic domain ${ }^{50}$. In contrast, ILEI selectively accelerated $\gamma$-secretase-independent degradation of APP-CTFs through a novel mechanism in which ILEI interacted with the PS1 $/ \gamma$-secretase complex but not APP on the extracellular or luminal side of the membrane. Modifier of cell adhesion, which is known as dedicator of cytokinesis 3 , a member of the dedicator of cytokinesis family of guanine nucleotide exchange factors, binds presenilins and selectively accelerates proteasome-mediated degradation of $\mathrm{APP}^{51}$. However, in contrast to ILEI, modifier of cell adhesion is a cytoplasmic protein that binds to presenilins on the cytoplasmic side of the membrane and destabilizes both APP-FL and APP-CTFs via an unknown mechanism ${ }^{52}$. Conversely, platelet-activating factor acetylhydrolase, isoform $1 \mathrm{~b}$, subunit 2 and sphingolipids inhibit $\gamma$-secretase-independent degradation of APP-CTFs and increase $\mathrm{A} \beta$ production ${ }^{53,54}$.

Previous studies have revealed a significant increase in APP-CTF $\beta$ as well as $A \beta$ in sporadic AD brains, which is sometimes associated with hyperactivity of $\beta$-secretase/BACE1 or downregulation of TGF- $\beta$ signalling ${ }^{5,6,31}$. In addition, the protein degradation activities of proteasomes, lysosomes and autophagy gradually decline with age and contribute to high levels of APP-CTF $\beta$ and $A \beta$, and consequently to an increased risk of sporadic $\mathrm{AD}^{7,8,45}$. On the other hand, clinical trials using non-selective inhibitors of $\gamma$-secretase activity were discontinued due to adverse effects that were probably caused by Notch inhibition and APP-CTF accumulation ${ }^{11-13,55}$. Thus, ILEI may be a plausible target for the development of diseasemodifying therapies for $\mathrm{AD}$, because ILEI may alter disease progression without perturbing Notch signalling and increasing APР-CTF $\beta$.

\section{Methods}

Plasmids. For construction of srPEN-2 (ref. 24), three silent nucleotide substitutions were introduced into human PEN-2 cDNA by PCR-based site-directed mutagenesis using the primer: $5^{\prime}$-GAACAGAGCCAAATCAAAGGTTACGTAT GGCGCTCAGCTGTGGGCTTCCTCTTC-3'. The pcDNA3-TAP plasmid containing DNA for the TAP tag, which consists of two immunoglobulin-binding domains of protein A from Staphylococcus aureus, a cleavage site for the tobacco etch virus (TEV) protease and the calmodulin-binding polypeptide, was obtained from Dr K. Oshikawa (Kyushu Universiy, Fukuoka, Japan). To generate pTAPsrPEN-2, the TAP tag fragment was fused in-frame to the $5^{\prime}$-end of srPEN-2 cDNA in pcDNA4 (Invitrogen, Carlsbad, CA, USA). A cDNA encoding ILEI with or without the stop codon was amplified from a human brain cDNA library (Clontech, San Diego, CA, USA) using PCR and was then ligated into pcDNA6/ V5-His (Invitrogen). To construct the ILEI knockdown vector, the oligonucleotide $5^{\prime}$-GGAGAAGUAUUAGACACU-3' was ligated into pSUP (Oligoengine, Seattle, WA, USA). To prepare an expression plasmid for siRNA-resistant ILEI, five neutral mutations were introduced into ILEI cDNA by PCR-based site-directed mutagenesis using the primer: 5'-GAAAAACAGGAGAGGTTCTGGATACTAA ATATTTTG-3'. The expression plasmids for PS1 mutants were constructed by PCR-based mutagenesis using the expression plasmid for wild-type human PS1 (ref. 56) as template. The sequences of all constructs were confirmed by sequencing. Notch $\Delta \mathrm{E}^{57}$ and HES-Y ${ }^{58}$ are gifts from Drs Raphael Kopan (Washington University, St Louis, MO, USA) and Masayasu Okochi (Osaka
} University, Osaka, Japan), respectively.
Identification of ILEI. To generate the HEK-TAP-PEN-2 and HEK-TAP empty cell lines, we transfected HEK293 cells (CRL-1573, ATCC) stably expressing PEN2-specific siRNA with pTAP-srPEN-2 or pTAP-empty. HEK-TAP-PEN-2 or HEK TAP-empty cells were homogenized in buffer A (20 mM Tris ( $\mathrm{pH} 7.5), 150 \mathrm{mM}$ $\mathrm{NaCl}, 0.5 \mathrm{mM}$ EDTA) containing a protease inhibitor cocktail (Roche Diagnostics, Mannheim, Germany). The postnuclear supernatants were centrifuged at 100,000 $\mathrm{g}$ for $1 \mathrm{~h}$ to collect the microsome membrane pellets. The pellet was solubilized in buffer A containing $1 \%$ CHAPSO and then mixed with IgG-sepharose beads followed by incubation at $4{ }^{\circ} \mathrm{C}$ for $2 \mathrm{~h}$. The mixture was washed with buffer $\mathrm{A}$ containing $0.5 \%$ CHAPSO. The beads were incubated with $10 \mathrm{U}$ of TEV protease in TEV buffer (10 mM Tris ( $\mathrm{pH} 8.0$ ), $150 \mathrm{mM} \mathrm{NaCl}, 0.5 \mathrm{mM}$ EDTA, 0.5\% CHAPSO) at $4{ }^{\circ} \mathrm{C}$ for $12 \mathrm{~h}$. After adjustment of the calcium concentration to $200 \mu \mathrm{M}$, the supernatant was mixed with calmodulin resin in three volumes of buffer $\mathrm{B}(10 \mathrm{mM}$ Tris (pH 8.0), $150 \mathrm{mM} \mathrm{NaCl}, 1 \mathrm{mM} \mathrm{Mg}$ acetate, $1 \mathrm{mM}$ imidazol, $2 \mathrm{mM} \mathrm{CaCl}_{2}, 0.5 \%$ CHAPSO) and then incubated for $5 \mathrm{~h}$ at $4{ }^{\circ} \mathrm{C}$. The proteins were eluted with SDS sample buffer and subjected to SDS-PAGE. The gel was stained with silver or SYPRO Ruby (Molecular Probes, Eugene, OR, USA). Bands were excised from the gel, and proteins in each gel piece that contained a specific band or a group of specific bands were digested with trypsin and subjected to liquid chromatographytandem mass spectrometry followed by a search of the Mascot database.

Immunoblotting analysis. The same amount of protein from cell lysates was loaded onto SDS-PAGE and transferred to a polyvinylidene fluoride membrane (Millipore, Billerica, MA, USA) or nitrocellurose membrane (BioRad, Hercules, CA, USA). The membranes were incubated with the following primary antibodies at $4{ }^{\circ} \mathrm{C}$ overnight, washed and incubated with corresponding horseradish peroxidase-conjugated secondary antibodies (1:3,500, Invitrogen) for $1 \mathrm{~h}$. Anti-human ILEI polyclonal antibody was raised in rabbits against a thyroglobulin-conjugated synthetic polypeptide corresponding to amino acid residues 126 to 143 with an added amino-terminal Cys residue (C+GGDVAPFIEFLKAIQDGT). This antibody was purified using immunoaffinity chromatography with immobilized antigen $(1: 4,000)$. The following antibodies were also used: goat polyclonal antiFAM3C antibody $(1: 2,000$, R\&D Systems, Minneapolis, MN, USA); rabbit polyclonal anti-NCT $(1: 5,000)$, anti-APP-CTF $(1: 10,000)$ and mouse monoclonal anti$\beta$-actin $(1: 10,000)$ antibodies (Sigma, St Louis, MO, USA); mouse monoclonal antiPS1 loop antibody (1:3,500, Chemicon, Temecula, CA, USA); rabbit polyclonal anti-APH-1L antibody (1:2,000, Covance, Princeton, NJ, USA); rabbit polyclonal anti-PEN-2 antibody (1:2,000, Calbiochem, San Diego, CA, USA); mouse monoclonal anti-Notch-1 antibody (mN1A, 1:1,000, AbD Serotec, Kidlington, UK); rabbit monoclonal anti-LRP1 antibody (1:5,000, Abcam, Cambridge, MA, USA); rabbit polyclonal anti-cadherin antibody (1:10,000, Sigma); mouse monoclonal antibody specific for the 17-26 amino acid residues of the human A $\beta$ (4G8, 1:1,000, Covance); rabbit polyclonal anti-neuron-specific enolase (1:10,000, Assay Biotechnology, Sunnyvale, CA, USA). Original immunoblots can be found in Supplementary Fig. 10.

Co-immunoprecipitation and chemical crosslinking. Cultured cells were lysed in a lysis buffer containing $1 \%$ CHAPSO. After pre-clearing with protein G-Sepharose 4 fast flow (GE Healthcare, Tokyo, Japan) for $1 \mathrm{~h}$, postnuclear supernatants were incubated with the appropriate antibody. The immunoprecipitates were recovered by overnight incubation with protein G-Sepharose and were analysed with immunoblotting. To eliminate IgG light-chain bands on immunoblots, signals were detected with horseradish peroxidase-conjugated bio-nanocapsules incorporating IgG Fc-binding $Z$ domains derived from $S$. aureus protein $\mathrm{A}^{59}$ (Beacle, Kyoto, Japan) instead of the secondary antibody. For crosslinking, HEK293 cells were treated with $2 \mathrm{mM}$ dithiobis succinimidyl propionate (Thermo Fisher Scientific, Kanagawa, Japan) for $2 \mathrm{~h}$ on ice. The reaction was stopped by adding Tris- $\mathrm{HCl}$ ( $\mathrm{pH} 7.5$, final $20 \mathrm{mM}$ ). After incubating on ice for $15 \mathrm{~min}$, the cells were lysed with a lysis buffer containing $1 \%$ Nonidet P-40. Under this condition, noncrosslinked components of the $\gamma$-secretase complex were reduced by $>80 \%$ on immunoblotting. After centrifugation at $12,000 \mathrm{~g}$, the supernatant was subjected to co-immunoprecipitation.

RNA interference. The following siRNA duplexes were purchased from Dhar macon (Lafayette, CO, USA): siGENOME SMART pool M-020514 for ILEI and D001210 for a non-targeting control. The M-020514 pool consisted of the four duplexes targeting the following sequences; F1: $5^{\prime}$-GAACAGCACAUAAAGAA CA-3'; F2: 5'-GGAGAAGUAUUAGACACUA-3'; F3: 5'-GGAGCACAUCUA UUACUAA- $3^{\prime}$; F4: 5' -GAACAAUAAGGAUACAAAC- $3^{\prime}$. Cultured cells were transfected with individual or pooled siRNA duplexes using Lipofectamine RNAi MAX (Invitrogen).

Secretase activity assays. For a cell-free $\gamma$-secretase assay, cultured cells were homogenized in HEPES buffer (25 mM HEPES ( $\mathrm{pH} 7.0$ ), $150 \mathrm{mM} \mathrm{NaCl}, 5 \mathrm{mM}$ $\mathrm{MgCl}_{2}, 5 \mathrm{mM} \mathrm{CaCl}_{2}$, a protease inhibitor cocktail) and the postnuclear supernatants were centrifuged at $100,000 \mathrm{~g}$ for $1 \mathrm{~h}$. The membrane pellets were lysed in $1 \%$ CHAPSO/HEPES buffer. Solubilized $\gamma$-secretase was recovered by centrifugation at $100,000 \mathrm{~g}$ for $30 \mathrm{~min}$, and the concentrations of protein and CHAPSO were adjusted to $0.25 \mathrm{mg} \mathrm{ml}^{-1}$ and $0.25 \%$, respectively. The resulting 
CHAPSO-solubilized $\gamma$-secretase was incubated with a recombinant APP-C99-Flag substrate for $6 \mathrm{~h}$ at $37^{\circ} \mathrm{C}$, and the $\mathrm{A} \beta 40$ and $\mathrm{A} \beta 42$ levels were measured by ELISA (WAKO Pure Chemical Industries, Osaka, Japan). For the NICD reporter assay, HEK293 cells in a 12-well plate were transiently transfected with Notch $\Delta \mathrm{E}$ (125 ng), HES-Y (125 ng) and $1.25 \mathrm{ng}$ of the control Renilla luciferase reporter plasmid pRL-TK (Promega, Osaka, Japan). The cells were lysed $24 \mathrm{~h}$ after transfection, and firefly and Renilla luciferase activities were quantified using a dual luciferase reporter assay system (Promega) and a luminometer AB-2250 (Atto, Tokyo, Japan). $\beta$-Secretase activity of cell lysates was measured using a fluorometric reaction based on $\beta$-secretase cleavage of a synthetic substrate (R\&D Systems).

Organotypic brain culture. Sagittal forebrain slices containing the hippocampus and cerebral cortex $(400-\mu \mathrm{m}$ thick) were prepared from 3-week-old female Wistar rats using a vibratome (Lancer Vibratome Series 1000, Vibratome, St Louis, MO, USA). The slices were cultured on semi-porous membrane inserts in six-well plates (0.4- $\mu \mathrm{m}$ pore diameter, Millipore) in a $37^{\circ} \mathrm{C}, 5 \% \mathrm{CO}_{2}, 99 \%$ humidity incubator. The slices were maintained in standard medium consisting of DMEM Ham's F12, $2 \%$ B27 neuronal supplement (Invitrogen) and antibiotic mixture $\left(5 \mu \mathrm{g} \mathrm{ml}^{-1}\right.$ penicillin, $5 \mu \mathrm{g} \mathrm{ml}^{-1}$ streptomycin and $10 \mu \mathrm{g} \mathrm{ml}^{-1}$ gentamycin). After $48 \mathrm{~h}$, slices were treated with $10 \mathrm{ng} \mathrm{ml}^{-1}$ TGF- $\beta 1$ (PeproTech, Rocky Hill, CT, USA) and rat ILEI-specific or non-targeting siRNA (Dharmacon) in Accell siRNA Delivery medium (Dharmacon) for another $48 \mathrm{~h}$.

Autopsy human brain tissues. Frozen brain tissues from the frontal and temporal cortices of 15 patients with $\mathrm{AD}, 15$ age-matched non-neurological disease control subjects and 10 non- $\mathrm{AD}$ neurological disease control subjects were obtained from the Brain Bank for Aging Research, Tokyo Metropolitan Institute of Gerontology (Tokyo, Japan). All the study subjects or their next of kin gave written informed consent for the brain donation, and the Shiga University of Medical Science Review Board approved the study protocol. All patients with AD fulfilled the National Institute of Neurological and Communicative Disorders and Stroke-Alzheimer's Disease and Related Disorders Associations criteria for probable AD.

Mice. The Tg vector pMoPrP-ILEI was constructed by subcloning an XhoI-XhoI fragment of human ILEI cDNA into the XhoI site of the MoPrP vector that contains the mouse prion promoter to achieve neuron-specific expression ${ }^{34}$. Tg mice were generated by injection of linearized pMoPrP-ILEI DNA into fertilized C57BL/6 mouse oocytes using standard techniques. Tg founders were identified using PCR (see below). Five founders from each injection were backcrossed to C57BL/6 mice for one to two generations before protein and morphology analysis, and the two lines with the highest expression levels were retained for further experiments. The genotypes of Tg mice were determined by PCR using the following transgene-specific primer pair; $\mathrm{mPrP}$-s $5^{\prime}$-CTGCTCCATTTTGC GTGACTC- $3^{\prime}$ and hFAM3C-as 5'-CTTCCAGGCAGATTTTGGGTC-3' . APP-Tg (Tg2576) mice $^{33}$ were obtained from Taconic Farms (Hudson, NY, USA) and were bred by mating male mice with ILEI-Tg females. Female littermates were used in this study unless otherwise stated. All animal experiments were performed in accordance with national guidelines (Ministry of Education, Culture, Sports, Science, and Technology) and approved by the Shiga University of Medical Science Institutional Animal Care and Use Committees.

Measurement of $\mathbf{A} \boldsymbol{\beta}$ in mouse brains. The right halves of mouse brains were homogenized using a motor-driven Teflon/glass homogenizer (ten strokes) in four volumes of Tris-buffered saline (20 mM Tris ( $\mathrm{pH} 7.5), 150 \mathrm{mM} \mathrm{NaCl}, 0.5 \mathrm{mM}$ EDTA) that contained a protease inhibitor cocktail. The homogenates were centrifuged at $100,000 \mathrm{~g}$ for $20 \mathrm{~min}$ on a TLA 100.4 rotor in a TLX ultracentrifuge (Beckman, Palo Alto, CA, USA). The supernatant was used as the soluble fraction. The pellet was lysed by brief sonication in an initial volume of $6 \mathrm{M}$ guanidine hydrochloride in $50 \mathrm{mM}$ Tris ( $\mathrm{pH} 7.5$ ), and then centrifuged at $100,000 \mathrm{~g}$ for $10 \mathrm{~min}$. The supernatant was diluted at 1:12 and used as the insoluble fraction. The soluble and insoluble fractions were subjected to a DC protein assay (BioRad) and ELISA assays specific for mouse/rat $A \beta 40$ and $A \beta 42$ (IBL, Gunma, Japan) or for human $A \beta 40$ and $A \beta 42$ (WAKO Pure Chemical Industries). For quantification of $A \beta$ plaque load on brain sections immunostained with anti-human $A \beta(1: 2,000$, IBL), the number of and percentage area occupied by cortical and hippocampal plaques were measured on digital pictures with the ImageJ 1.46 program (National Institutes of Health, Bethesda, MD, USA).

Immunohistochemistry. The left halves of mouse brains were fixed in $4 \%$ paraformaldehyde in phosphate buffer. After blocking endogenous peroxidase activity, free-floating brain sections were incubated with appropriately diluted primary antibodies in PBS containing $2 \%$ BSA and $0.3 \%$ Triton-X100 at $4{ }^{\circ} \mathrm{C}$ overnight under horizontal agitation. Sections were then incubated with biotinylated secondary antibodies for $1 \mathrm{~h}$. The immunoreactive products were visualized by incubating with $3,3^{\prime}$-diaminobenzidine containing nickel ammonium sulphate as an enhancing reagent. Stained sections were observed using a microscope (Olympus BX50, Tokyo, Japan). For double fluorescent immunostaining, sections were incubated with a mixture of rabbit polyclonal anti-ILEI antibody $(1: 4,000)$ and mouse monoclonal antibody against non-phosphorylated neurofilament (1:4,000, Covance), glial fibrillary acidic protein (1:4,000, Dako, Tokyo, Japan) or Ibal $\left(1: 2,000\right.$, WAKO Pure Chemical Industries) overnight at $4{ }^{\circ} \mathrm{C}$, then reacted with a mixture of secondary antibodies conjugated with Alexa488 (1:500, green) and Alexa594 (1:500, red) (Molecular Probes). The stained sections were analysed using a confocal laser-scanning microscope system (Digital Eclipse C1si-Ready, Nikon, Tokyo, Japan).

Y-maze test. The Y-maze apparatus consisted of three arms with grey walls ( $40 \mathrm{~cm}$ long, $10 \mathrm{~cm}$ wide, $10 \mathrm{~cm}$ high). The insides of the arms were identical, providing no intramaze cues. Each mouse was placed in the centre of the symmetrical Y-maze and was allowed to explore the maze freely for $8 \mathrm{~min}$. The number and sequence of arms entered were recorded manually. The total number of arm entries was used to measure locomotor activity and motivation to explore the maze The percentage of alternation, which was calculated by the proportion of alternations (an arm choice differing from the previous two choices) to the total number of alternation opportunities (total arm entries minus two), was used as a measure of spatial working memory. Experiments were done blind regarding the genotype of the mice.

Statistical analysis. Statistical evaluation was performed using two-tailed unpaired Student's $t$-test unless otherwise stated. Data are presented as means \pm s.d. Statistical significance was defined at ${ }^{*} P<0.05$ or ${ }^{*} P<0.01$.

\section{References}

1. De Strooper, B., Iwatsubo, T. \& Wolfe, M. S. Presenilins and $\gamma$-secretase: structure, function, and role in Alzheimer disease. Cold Spring Harb. Perspect. Med. 2, a006304 (2012).

2. Muller, U. C. \& Zheng, H. Physiological functions of APP family proteins. Cold Spring Harb. Perspect. Med. 2, a006288 (2012).

3. Ring, S. et al. The secreted $\beta$-amyloid precursor protein ectodomain APPs $\alpha$ is sufficient to rescue the anatomical, behavioral, and electrophysiological abnormalities of APP-deficient mice. J. Neurosci. 27, 7817-7826 (2007)

4. St George-Hyslop, P. H. \& Petit, A. Molecular biology and genetics of Alzheimer's disease. C. R. Biol. 328, 119-130 (2005).

5. Fukumoto, H., Cheung, B. S., Hyman, B. T. \& Irizarry, M. C. $\beta$-secretase protein and activity are increased in the neocortex in Alzheimer disease. Arch. Neurol. 59, 1381-1389 (2002).

6. Yang, L. B. et al. Elevated $\beta$-secretase expression and enzymatic activity detected in sporadic Alzheimer disease. Nat. Med. 9, 3-4 (2003).

7. Riederer, B. M., Leuba, G., Vernay, A. \& Riederer, I. M. The role of the ubiquitin proteasome system in Alzheimer's disease. Exp. Biol. Med. (Maywood) 236, 268-276 (2011).

8. Nixon, R. A. \& Yang, D. S. Autophagy failure in Alzheimer's disease--locating the primary defect. Neurobiol. Dis. 43, 38-45 (2011).

9. Chang, K. A. \& Suh, Y. H. Pathophysiological roles of amyloidogenic carboxyterminal fragments of the $\beta$-amyloid precursor protein in Alzheimer's disease. J. Pharmacol. Sci. 97, 461-471 (2005).

10. Bittner, T. et al. $\gamma$-secretase inhibition reduces spine density in vivo via an amyloid precursor protein-dependent pathway. J. Neurosci. 29, 10405-10409 (2009).

11. Wong, G. T. et al. Chronic treatment with the $\gamma$-secretase inhibitor LY-411,575 inhibits $\beta$-amyloid peptide production and alters lymphopoiesis and intestinal cell differentiation. J. Biol. Chem. 279, 12876-12882 (2004).

12. Mitani, Y. et al. Differential effects between $\gamma$-secretase inhibitors and modulators on cognitive function in amyloid precursor protein-transgenic and nontransgenic mice. J. Neurosci. 32, 2037-2050 (2012).

13. Doody, R. S. et al. A phase 3 trial of semagacestat for treatment of Alzheimer's disease. N. Engl. J. Med. 369, 341-350 (2013).

14. De Strooper, B. \& Annaert, W. Novel research horizons for presenilins and $\gamma$ secretases in cell biology and disease. Annu. Rev. Cell. Dev. Biol. 26, 235-260 (2010).

15. Mitsuishi, Y. et al. Human CRB2 inhibits $\gamma$-secretase cleavage of amyloid precursor protein by binding to the presenilin complex. J. Biol. Chem. 285, 14920-14931 (2010).

16. Hasegawa, H., Liu, L. \& Nishimura, M. Dilysine retrieval signal-containing p24 proteins collaborate in inhibiting $\gamma$-cleavage of amyloid precursor protein. J. Neurochem. 115, 771-781 (2010).

17. He, G. et al. $\gamma$-secretase activating protein is a therapeutic target for Alzheimer's disease. Nature 467, 95-98 (2010).

18. Chen, F. et al. TMP21 is a presenilin complex component that modulates $\gamma$-secretase but not $\varepsilon$-secretase activity. Nature 440, 1208-1212 (2006).

19. Wakabayashi, T. et al. Analysis of the $\gamma$-secretase interactome and validation of its association with tetraspanin-enriched microdomains. Nat. Cell. Biol. 11, 1340-1346 (2009). 
20. Rigaut, G. et al. A generic protein purification method for protein complex characterization and proteome exploration. Nat. Biotechnol. 17, 1030-1032 (1999).

21. Esler, W. P. et al. Activity-dependent isolation of the presenilin- $\gamma$-secretase complex reveals nicastrin and a $\gamma$ substrate. Proc. Natl Acad. Sci. USA 99, 2720-2725 (2002).

22. Zhu, Y. et al. Cloning, expression, and initial characterization of a novel cytokine-like gene family. Genomics 80, 144-150 (2002).

23. Zhao, G., Liu, Z., Ilagan, M. X. \& Kopan, R. $\gamma$-secretase composed of PS1/Pen2/ Aphla can cleave Notch and amyloid precursor protein in the absence of nicastrin. J. Neurosci. 30, 1648-1656 (2010).

24. Hasegawa, H. et al. Both the sequence and length of the C terminus of PEN-2 are critical for intermolecular interactions and function of presenilin complexes. J. Biol. Chem. 279, 46455-46463 (2004).

25. Pitsi, D. \& Octave, J. N. Presenilin 1 stabilizes the C-terminal fragment of the amyloid precursor protein independently of $\gamma$-secretase activity. J. Biol. Chem. 279, 25333-25338 (2004).

26. Watanabe, N. et al. Functional analysis of the transmembrane domains of presenilin 1: participation of transmembrane domains 2 and 6 in the formation of initial substrate-binding site of $\gamma$-secretase. J. Biol. Chem. 285, 19738-19746 (2010)

27. Herreman, A. et al. Total inactivation of $\gamma$-secretase activity in presenilindeficient embryonic stem cells. Nat. Cell Biol. 2, 461-462 (2000).

28. Waerner, T. et al. ILEI: a cytokine essential for EMT, tumor formation, and late events in metastasis in epithelial cells. Cancer Cell 10, 227-239 (2006).

29. Xia, W. et al. Presenilin complexes with the C-terminal fragments of amyloid precursor protein at the sites of amyloid $\beta$-protein generation. Proc. Natl Acad. Sci. USA 97, 9299-9304 (2000).

30. Choy, R. W., Cheng, Z. \& Schekman, R. Amyloid precursor protein (APP) traffics from the cell surface via endosomes for amyloid $\beta$ (A $\beta$ ) production in the trans-Golgi network. Proc. Natl Acad. Sci. USA 109, E2077-E2082 (2012).

31. Tesseur, I. et al. Deficiency in neuronal TGF- $\beta$ signaling promotes neurodegeneration and Alzheimer's pathology. J. Clin. Invest. 116, 3060-3069 (2006).

32. Chaudhury, A. et al. TGF- $\beta$-mediated phosphorylation of hnRNP E1 induces EMT via transcript-selective translational induction of Dab2 and ILEI. Nat. Cell Biol. 12, 286-293 (2010).

33. Hsiao, K. et al. Correlative memory deficits, $A \beta$ elevation, and amyloid plaques in transgenic mice. Science 274, 99-102 (1996).

34. Borchelt, D. R. et al. A vector for expressing foreign genes in the brains and hearts of transgenic mice. Genet. Anal. 13, 159-163 (1996).

35. Johansson, P. et al. FAM3B PANDER and FAM3C ILEI represent a distinct class of signaling molecules with a non-cytokine-like fold. Structure 21, 306-313 (2013)

36. Lahsnig, C. et al. ILEI requires oncogenic Ras for the epithelial to mesenchymal transition of hepatocytes and liver carcinoma progression. Oncogene $\mathbf{2 8}$, 638-650 (2009)

37. Wilson, C. G., Robert-Cooperman, C. E. \& Burkhardt, B. R. PANcreaticDERived factor: novel hormone PANDERing to glucose regulation. FEBS Lett. 585, 2137-2143 (2011).

38. Katahira, T., Nakagiri, S., Terada, K. \& Furukawa, T. Secreted factor FAM3C (ILEI) is involved in retinal laminar formation. Biochem. Biophys. Res. Commun. 392, 301-306 (2010).

39. Caraci, F. et al. TGF- $\beta 1$ pathway as a new target for neuroprotection in Alzheimer's disease. CNS Neurosci. Ther. 17, 237-249 (2011).

40. Caraci, F. et al. The CC genotype of transforming growth factor- $\beta 1$ increases the risk of late-onset Alzheimer's disease and is associated with AD-related depression. Eur. Neuropsychopharmacol. 22, 281-289 (2012).

41. Xiao, F. et al. Proteomic analysis of cerebrospinal fluid from patients with idiopathic temporal lobe epilepsy. Brain Res. 1255, 180-189 (2009).

42. Mackenzie, I. R. \& Miller, L. A. Senile plaques in temporal lobe epilepsy. Acta. Neuropathol. 87, 504-510 (1994).

43. Davies, H., Blennow, K., McGuire, J., Podust, V. \& Simonsen, A. Saponin D and FAM3C are biomarkers for Alzheimer's disease. US Patent 7993868 (2011).

44. Nunan, J. et al. The C-terminal fragment of the Alzheimer's disease amyloid protein precursor is degraded by a proteasome-dependent mechanism distinct from $\gamma$-secretase. Eur. J. Biochem. 268, 5329-5336 (2001).

45. Flood, F. et al. Proteasome-mediated effects on amyloid precursor protein processing at the $\gamma$-secretase site. Biochem. J. 385, 545-550 (2005).
46. Caporaso, G. L., Gandy, S. E., Buxbaum, J. D. \& Greengard, P. Chloroquine inhibits intracellular degradation but not secretion of Alzheimer $\beta / \mathrm{A} 4$ amyloid precursor protein. Proc. Natl Acad. Sci. USA 89, 2252-2256 (1992).

47. Jaeger, P. A. et al. Regulation of amyloid precursor protein processing by the Beclin 1 complex. PLoS ONE 5, e11102 (2010).

48. Lai, M. T. et al. Presenilin-1 and presenilin-2 exhibit distinct yet overlapping $\gamma$ secretase activities. J. Biol. Chem. 278, 22475-22481 (2003).

49. Sato, C., Takagi, S., Tomita, T. \& Iwatsubo, T. The C-terminal PAL motif and transmembrane domain 9 of presenilin 1 are involved in the formation of the catalytic pore of the $\gamma$-secretase. J. Neurosci. 28, 6264-6271 (2008).

50. Suzuki, T. \& Nakaya, T. Regulation of amyloid $\beta$-protein precursor by phosphorylation and protein interactions. J. Biol. Chem. 283, 29633-29637 (2008).

51. Chen, Q., Kimura, H. \& Schubert, D. A novel mechanism for the regulation of amyloid precursor protein metabolism. J. Cell Biol. 158, 79-89 (2002).

52. Kashiwa, A. et al. Isolation and characterization of novel presenilin binding protein. J. Neurochem. 75, 109-116 (2000).

53. Page, R. M. et al. Loss of PAFAH1B2 reduces amyloid- $\beta$ generation by promoting the degradation of amyloid precursor protein $\mathrm{C}$-terminal fragments. J. Neurosci. 32, 18204-18214 (2012).

54. Tamboli, I. Y. et al. Sphingolipid storage affects autophagic metabolism of the amyloid precursor protein and promotes A $\beta$ generation. J. Neurosci. 31, 1837-1849 (2011).

55. Chavez-Gutierrez, L. et al. The mechanism of $\gamma$-secretase dysfunction in familial Alzheimer disease. EMBO J. 31, 2261-2274 (2012).

56. Nishimura, M. et al. Presenilin mutations associated with Alzheimer disease cause defective intracellular trafficking of $\beta$-catenin, a component of the presenilin protein complex. Nat. Med. 5, 164-169 (1999).

57. Schroeter, E. H., Kisslinger, J. A. \& Kopan, R. Notch-1 signalling requires ligand-induced proteolytic release of intracellular domain. Nature 393, 382-386 (1998).

58. Tagami, S. et al. Regulation of Notch signaling by dynamic changes in the precision of S3 cleavage of Notch-1. Mol. Cell Biol. 28, 165-176 (2008).

59. Iijima, M. et al. Nanocapsules incorporating IgG Fc-binding domain derived from Staphylococcus aureus protein A for displaying IgGs on immunosensor chips. Biomaterials 32, 1455-1464 (2011).

60. Edbauer, D., Willem, M., Lammich, S., Steiner, H. \& Haass, C. Insulindegrading enzyme rapidly removes the $\beta$-amyloid precursor protein intracellular domain (AICD). J. Biol. Chem. 277, 13389-13393 (2002).

\section{Acknowledgements}

This work was supported in part by Grants-in-Aid for Scientific Research from the Ministry of Education, Culture, Sports, Science, and Technology, Japan (23500445 to M.N. and 23590359 to H.H.), a grant from the A-STEP, Japan Science and Technology Agency (AS231Z00919G to M.N.) and a grant from the Program for the Promotion of Fundamental Studies in Health Sciences of the National Institute of Biomedical Innovation, Japan (05-26 to M.N.).

\section{Author contributions}

H.H. and L.L. performed the TAP and cell culture experiments. L.L. contributed to the organotypic culture and immunohistochemistry experiments. L.L., I.T. and M.N. carried out the Tg mouse experiments. S.M. collected autopsy brain samples and performed neuropathological diagnosis. M.N. designed the study, supervised all of the experiments and wrote the manuscript.

\section{Additional information}

Supplementary Information accompanies this paper at http://www.nature.com/ naturecommunications

Competing financial interests: The authors declare no competing financial interests.

Reprints and permission information is available online at http://npg.nature.com/ reprintsandpermissions/

How to cite this article: Hasegawa, H. et al. The FAM3 superfamily member ILEI ameliorates Alzheimer's disease-like pathology by destabilizing the penultimate amyloid$\beta$ precursor. Nat. Commun. 5:3917 doi: 10.1038/ncomms4917 (2014). 